\title{
Outreach strategies for expanding health insurance coverage in children (Review)
}

\author{
Meng Q, Yuan B, Jia L, Wang J, Garner P
}

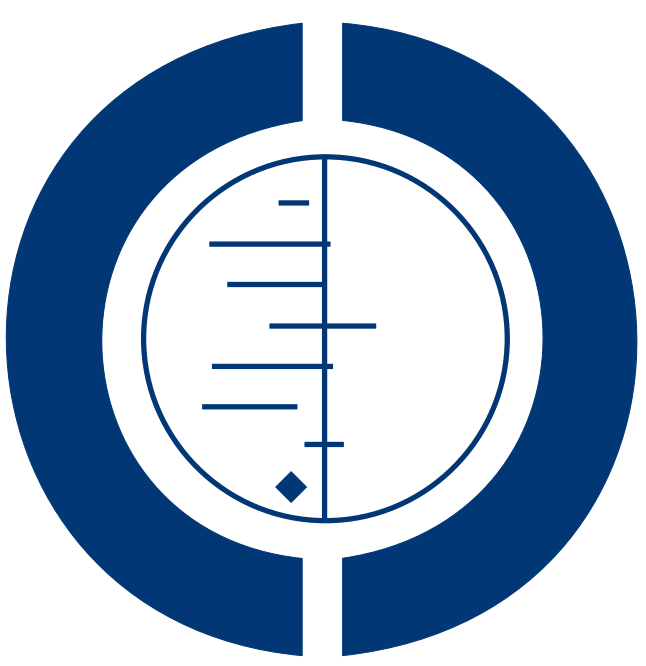

\section{THE COCHRANE COLLABORATION $^{\circledR}$}

This is a reprint of a Cochrane review, prepared and maintained by The Cochrane Collaboration and published in The Cochrane Library 2010, Issue 8

http://www.thecochranelibrary.com

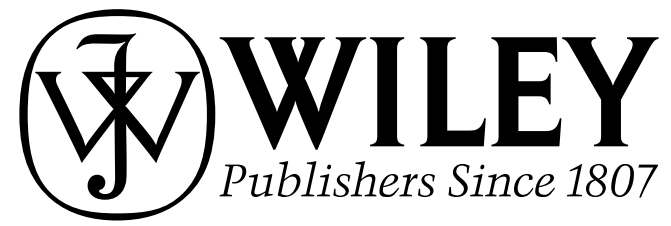

Outreach strategies for expanding health insurance coverage in children (Review)

Copyright $\odot 2010$ The Cochrane Collaboration. Published by John Wiley \& Sons, Ltd. 
TABLE OF CONTENTS

HEADER . . . . . . . . . . . . . . . . . . . . . . . . . . . . . . . . . . . . . . . 1

ABSTRACT . . . . . . . . . . . . . . . . . . . . . . . . . . . . . . . . . . . . . . . . . . . . . 1

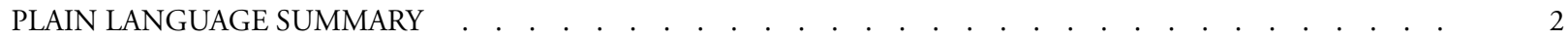

SUMMARY OF FINDINGS FOR THE MAIN COMPARISON . . . . . . . . . . . . . . . . . . . . 2

BACKGROUND . . . . . . . . . . . . . . . . . . . . . . . . . . . . . . . . . . . . 5

OBJECTIVES . . . . . . . . . . . . . . . . . . . . . . . . . . . . . . . . . . . . . 6

METHODS . . . . . . . . . . . . . . . . . . . . . . . . . . . . . . . . . . . . . . 6

RESULTS . . . . . . . . . . . . . . . . . . . . . . . . . . . . . . . . . . . . . . . 9

Figure 1. . . . . . . . . . . . . . . . . . . . . . . . . . . . . . . . . . . . . . 10

Figure 2. . . . . . . . . . . . . . . . . . . . . . . . . . . . . . . . . . . . . . 11

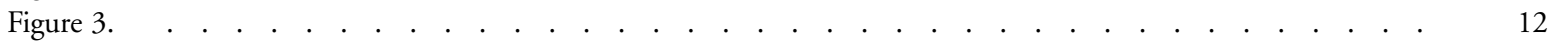

ADDITIONAL SUMMARY OF FINDINGS . . . . . . . . . . . . . . . . . . . . . . . . . . . . . . . $\quad . \quad 13$

DISCUSSION . . . . . . . . . . . . . . . . . . . . . . . . . . . . . . . . . . . . . 15

AUTHORS' CONCLUSIONS . . . . . . . . . . . . . . . . . . . . . . . . . . . . . . . . . . . 15

ACKNOWLEDGEMENTS . . . . . . . . . . . . . . . . . . . . . . . . . . . . . . . . . . . . . . . . .

REFERENCES . . . . . . . . . . . . . . . . . . . . . . . . . . . . . . . . . . . . . 16

CHARACTERISTICS OF STUDIES . . . . . . . . . . . . . . . . . . . . . . . . . . . . . . . . . 18

DATA AND ANALYSES . . . . . . . . . . . . . . . . . . . . . . . . . . . . . . . . . . . . . . . . . . . . . . . . . . . . $\quad 24$

Analysis 1.1. Comparison 1 Awareness and application support versus no intervention, Outcome 1 Enrolment. $\quad . \quad$. 24

Analysis 1.2. Comparison 1 Awareness and application support versus no intervention, Outcome 2 Continuous enrolment. . . . . . . . . . . . . . . . . . . . . . . . . . . . . . . . . . . . 25

Analysis 1.3. Comparison 1 Awareness and application support versus no intervention, Outcome 3 Sporadic enrolment. 25

Analysis 1.4. Comparison 1 Awareness and application support versus no intervention, Outcome 4 Mean time to obtain insurance. . . . . . . . . . . . . . . . . . . . . . . . . . . . . . . . . . . . 26

Analysis 1.5. Comparison 1 Awareness and application support versus no intervention, Outcome 5 Parental satisfaction with process of enrolment. . . . . . . . . . . . . . . . . . . . . . . . . . . . . . 26

Analysis 2.1. Comparison 2 Handing out applications versus no intervention, Outcome 1 Enrolment. . . . . . . 27

APPENDICES . . . . . . . . . . . . . . . . . . . . . . . . . . . . . . . . . . . . . 27

HISTORY . . . . . . . . . . . . . . . . . . . . . . . . . . . . . . . . . . . . . . . 41

CONTRIBUTIONS OF AUTHORS . . . . . . . . . . . . . . . . . . . . . . . . . . . . . . . . . . . . . . 41

DECLARATIONS OF INTEREST . . . . . . . . . . . . . . . . . . . . . . . . . . . . . . . . . . . . . . 41

SOURCES OF SUPPORT . . . . . . . . . . . . . . . . . . . . . . . . . . . . . . . . . . . . . . . . . 41

DIFFERENCES BETWEEN PROTOCOL AND REVIEW . . . . . . . . . . . . . . . . . . . . . . . . . 41

INDEX TERMS . . . . . . . . . . . . . . . . . . . . . . . . . . . . . . . . . . . . . . 41

Outreach strategies for expanding health insurance coverage in children (Review)

Copyright $(2010$ The Cochrane Collaboration. Published by John Wiley \& Sons, Ltd. 


\title{
[Intervention Review]
}

\section{Outreach strategies for expanding health insurance coverage in children}

\author{
Qingyue Meng ${ }^{1}$, Beibei Yuan ${ }^{1}$, Liying Jia ${ }^{1}$, Jian Wang ${ }^{1}$, Paul Garner ${ }^{2}$ \\ ${ }^{1}$ Center for Health Management and Policy, Shandong University, Jinan, China. ${ }^{2}$ International Health Group, Liverpool School of \\ Tropical Medicine, Liverpool, UK \\ Contact address: Qingyue Meng, Center for Health Management and Policy, Shandong University, Wenhua Xi Road 44, Jinan, \\ Shandong, 250012, China. qmeng@sdu.edu.cn.
}

Editorial group: Cochrane Effective Practice and Organisation of Care Group.

Publication status and date: New, published in Issue 8, 2010.

Review content assessed as up-to-date: 4 July 2010.

Citation: Meng Q, Yuan B, Jia L, Wang J, Garner P. Outreach strategies for expanding health insurance coverage in children. Cochrane Database of Systematic Reviews 2010, Issue 8. Art. No.: CD008194. DOI: 10.1002/14651858.CD008194.pub2.

Copyright (C) 2010 The Cochrane Collaboration. Published by John Wiley \& Sons, Ltd.

\begin{abstract}
A B S T R A C T
Background

Health insurance has the potential to improve access to health care and protect people from healthcare costs when they are ill. However, coverage is often low, particularly in people most in need of protection.
\end{abstract}

\section{Objectives}

To assess the effectiveness of outreach strategies for expanding insurance coverage of children who are eligible for health insurance schemes.

\section{Search strategy}

We searched the Cochrane Effective Practice and Organisation of Care Group (EPOC) Specialised Register (The Cochrane Library 2009, Issue 2), PubMed (January 1951 to January 2010), EMBASE (January 1966 to April 2009), PsycINFO (January 1967 to April 2009) and other relevant databases and websites. In addition, we searched the reference lists of included studies and relevant reviews, and carried out a citation search for included studies to find more potentially relevant studies.

\section{Selection criteria}

Randomised controlled trials, controlled clinical trials, controlled before-after studies and interrupted time series which evaluated the effects of outreach strategies on increasing health insurance coverage for children. We defined outreach strategies as measures to improve the implementation of existing health insurance to enrol more eligible populations. This included increasing awareness of schemes, modifying enrolment, improving management and organis ation of insurance schemes, and mixed strategies.

Data collection and analysis

Two review authors independently extracted data and assessed the risk of bias. We narratively summari sed the data.

\section{Main results}

We included two studies, both from the United States. One randomised controlled trial study with a low risk of bias showed that community- based case managers who provided health insurance information, application support, and negotiated with the insurer were effective in enrolling and maintaining enrolment of Latino American children into health insurance schemes $(\mathrm{n}=257)$. The second quasi-randomised controlled trial, with an unclear risk of bias $(n=223)$, indicated that handing out insurance application materials in hospital emergenc y departments can increase enrolment of children into health insurance. 


\section{Authors' conclusions}

The two studies included in this review provide evidence that in the US providing health insurance information and application assistance, and handing out application materials in hospital emergency departments can probably both improve insurance coverage of children. Further studies evaluating the effectiveness of different outreach strategies for expanding health insurance coverage of children in different countries are needed, with careful attention given to study design.

\section{PLAIN LANGUAGE SUMMARY}

\section{Measures to increase health insurance coverage in children}

This review assesses the effectiveness of outreach strategies to increase health insurance coverage in children and includes two studies. One trial evaluated case managers who provide health insurance information and application assistance; a second trial evaluated simply handing out application materials in emergency departments of hospitals. Both measures appeared to be effective in increasing insurance enrolment of children in the US. We found no reliable studies evaluating other outreach strategies or studies from other countries. 


\begin{tabular}{|c|c|c|c|c|c|}
\hline SUMMARY OF & F I N D I N G & MAIN & COMPAF & I S O N [Explanation] & \\
\hline Awareness and application su & upport compared & with no intervention & & & \\
\hline $\begin{array}{l}\text { Patient or population: Latino A } \\
\text { Settings: Developed country, } \\
\text { Intervention: Awareness and } \\
\text { Comparison: No intervention }\end{array}$ & $\begin{array}{l}\text { American children } \\
\text { district level } \\
\text { application suppo }\end{array}$ & $\begin{array}{l}\text { n with no health insurance covera } \\
\text { ort }\end{array}$ & & & \\
\hline Outcomes & NO. of studies & Relative effect & NO. of participants & $\begin{array}{l}\text { Quality of the evidence } \\
\text { (GRADE) }\end{array}$ & Comments \\
\hline Enrolment into insurance & 1 & RR 1.68 (95\% Cl 1.44 to 1.96$)$ & 257 & $\begin{array}{l}+++0 \\
\text { moderate [1] }\end{array}$ & $\begin{array}{l}\text { RR calculation is based on the number of } \\
\text { events imputed from percentage and the num- } \\
\text { ber of participants measured on correspond- } \\
\text { ing outcome }\end{array}$ \\
\hline Continuous enrolment & 1 & RR 2.59 (95\% Cl 1.95 to 3.44$)$ & 257 & $\begin{array}{l}+++0 \\
\text { moderate [1] }\end{array}$ & See comment above \\
\hline Sporadic enrolment & 1 & RR 0.66 (95\% $\mathrm{Cl} 0.41$ to 1.05$)$ & 257 & $\begin{array}{l}+++0 \\
\text { moderate }[1]\end{array}$ & See comment above \\
\hline Mean time to obtain insurance & 1 & $\begin{array}{l}\mathrm{MD}-47.30(95 \% \mathrm{Cl}-73.98 \text { to }- \\
20.62)\end{array}$ & 200 & $\begin{array}{l}+++0 \\
\text { moderate [1] }\end{array}$ & $\begin{array}{l}\text { MD calculation is based on mean, SD and } \\
\text { the number of participants measured on this } \\
\text { outcome }\end{array}$ \\
\hline $\begin{array}{l}\text { Parental satisfaction with pro- } \\
\text { cess of enrolment }\end{array}$ & 1 & $\mathrm{MD}-1.07(95 \% \mathrm{Cl}-1.42$ to -0.72$)$ & 173 & $\begin{array}{l}+++0 \\
\text { moderate [1] }\end{array}$ & $\begin{array}{l}\text { MD calculation is based on mean, SD and } \\
\text { the number of participants measured on this } \\
\text { outcome } \\
\text { Parental satisfaction score was examined with } \\
\text { Likert scale scores where } 1=\text { very satisfied, } \\
2=\text { satisfied, } 3=\text { uncertain, } 4=\text { dissatisfied, } \\
5=\text { very dissatisfied }\end{array}$ \\
\hline
\end{tabular}


GRADE Working Group grades of evidence

High quality: Further research is very unlikely to change our confidence in the estimate of effect.

Moderate quality: Further research is likely to have an important impact on our confidence in the estimate of effect and may change the estimate.

Low quality: Further research is very likely to have an important impact on our confidence in the estimate of effect and is likely to change the estimate.

very low quality: We are very uncertain about the estimate.

FOOTNOTES:

[1] One RCT study (Flores 2005) evaluating this intervention. This RCT has a low risk of bias in design and implementation, but only targeted Latino American children, and this intervention involved a specially trained case manager, which downgrades the evidence regarding each outcome. 


\section{B A C K G R O U N D}

\section{Description of the condition}

Health insurance can improve access to health care for the insured population and protect them from the burden of unexpected healthcare costs. For example, a study found that uninsured children in the US were less likely to have a regular source of primary health care, and that they used medical and dental care less often (Institute of Medicine 2002). Studies from China and the US have revealed that immunisation of children was positively associated with coverage of health insurance schemes (Arnold 1992; Zhang 1999). Some empirical studies in the US have also shown that poor health status in children is associated with being uninsured (Holl 1995; Kuehl 2000; Li 2001).

However, for most developing countries and some developed countries which have not achieved universal coverage of health insurance by a tax-based system or social health insurance, lack of health insurance coverage is still both a social concern and a policy issue. In these countries, the particular groups at risk are children, the elderly, women, low-income populations, rural populations, racial/ethnic minorities and immigrants - all of whom are at higher risk of health problems, and all more likely to be uninsured. In this review, we limited our focus to children, one of the groups most likely to lack health insurance. The figures in the United States indicate that the percentage of children without health insurance rose from 10.8\% in 2004 to $11.2 \%$ in 2005 (DeNavas-Walt 2006). In China, a survey in Shanghai in 2006 found that the uninsured rate of children whose parents had migrated in from rural areas was $65.6 \%$ compared to $21.2 \%$ in children of permanent residents, and in both groups children from low-income families were more likely to be uninsured ( $\mathrm{Lu}$ 2008).

To explore strategies that have been used to expand coverage, we took all available literature concerning countries that had already a health insurance programme in place, but were planning to expand it to vulnerable populations, including children, the elderly, women, low-income individuals, rural populations, racial/ethnic minorities, immigrants, populations with chronic disease and populations with disability. We mapped out and carefully documented all the strategies used to increase coverage, and the categories we identified are outlined in Table 1. These strategies can be divided into two main categories: a) strategies adopted during design; and b) strategies added on to programme implementation.

Table 1. Strategies to expand health insurance

\begin{tabular}{l|l}
\hline Strategies & Contents \\
\hline Improving design of health insurance schemes & $\begin{array}{l}\text { - Modifying the eligibility criteria } \\
\text { - Making the premium affordable } \\
\text { - Improving health care delivery }\end{array}$ \\
\hline $\begin{array}{ll}\text { Improving implementation of insurance schemes (outreach strate- } \\
\text { gies) }\end{array}$ & $\begin{array}{l}\text { - Increasing awareness of schemes and benefits } \\
\text { - Modifying enrolment } \\
\text { - Improving management and organization of insurance } \\
\text { schemes }\end{array}$ \\
\hline
\end{tabular}

For those adopted during design, the purpose is to improve the design of health insurance schemes by measures such as modifying eligibility criteria to include more children, setting an appropriate premium level or providing subsidies, or improving the content, price and quality of the health care package.

However, even if a health insurance scheme is well-designed, it does not mean that all eligible children will join it. For example, more than $60 \%$ of uninsured children were already eligible for Medicaid or the State Children Health Insurance Program (SCHIP) in 2002 (Jacobson 2007). Thus strategies that specifically aim to improve enrolment into existing insurance schemes are very important. Some researchers have explored the barriers to enrolment of potentially eligible children, for example awareness, financial, administrative and geographic barriers. An interview about chil- dren's enrolment in the US SCHIP found that some members of indigenous groups did not know about SCHIP or, if they did know, were still concerned about the potential costs associated with enrolment in these programmes, because they were not aware of their eligibility for exemption from premiums and cost-sharing requirements (Langwell 2003). The complexity of the application process can also be a barrier. A survey of parents indicated that although $93.5 \%$ had heard of the insurance programmes for their children, only $48.1 \%$ thought the application processes were easy (Haley 2007). In addition, the requirement for applicants for health insurance to travel to enrolment sites has also proved to be a constraint for families in rural or isolated places (Langwell 2003). A variety of approaches have been used to improve implementa- 
tion of insurance scheme and increase the enrolment of eligible children. These strategies include increasing awareness about the scheme, or making the application process easier and more userfriendly. For example, Californiass advertising campaign via television, radio and print in 48 counties was implemented in both English and Spanish to inform more eligible children about insurance schemes (Aizer 2006). Georgia State shortened its application form in 1993, and saw a 42\% increase in enrolment among pregnant women and children (Que 1998). The community health insurance in Uganda linked the time of collecting premiums with a suitable event in the community, like burial societies' monthly meetings, which facilitated either enrolment of first-time members or renewal of membership (Carrin 2003). In this review, all of these approaches are defined as outreach strategies.

This review assesses the effectiveness of outreach strategies that improve implementation of existing schemes to expand enrolment. It does not look at how the design of insurance schemes could influence coverage.

\section{Description of the intervention}

Outreach strategies in this review are defined as measures to improve the implementation of existing health insurance to cover its eligible population, which aim to maximise the enrolment rate of health insurance without changing the content of insurance (eligible population, premium, benefit package). These strategies can be mainly grouped into the following categories (Meng 2010).

- Increasing awareness of schemes: this includes information distribution via television or radio advertisements, telephone hotlines, or through schools or other particular locations to raise awareness of insurance schemes. For example, many states in the US distribute information about SCHIP in venues where target parents and children tend to congregate, including early childhood centres, schools, hospitals and religious institutions (Andrulis 1999).

- Modifying enrolment: this refers to any methods of improving or simplifying the enrolment procedures, including programmes that help families with the paperwork and other aspects of application; positioning eligibility workers in schools or health facilities; authorising more entities to interview applicants; shared eligibility with other insurance or public programmes. For example, the state government of California worked with community-based organisations to provide application assistance to families who were potentially eligible for Healthy Families (California SCHIP) or Medicaid (Buchmueller 2007). Florida's Healthy Start Program has linked eligibility for Medicaid through the school lunch programme, in which children eligible for the school lunch programme are automatically eligible for health insurance (Secretary of Health and Human Services 1998).

- Improving management and organisation of insurance schemes: this refers to strategies that aim to improve the capacity of insurers to manage the insurance schemes, including better information systems, and training staff for better and more effective outreach and management. For example, in order to enrol Latino children, many states in the US have supported staff development and training in cultural competency to include appropriate family members (Zambrana 2004).

\section{Why it is important to do this review}

Many outreach strategies have been implemented and evaluated. A critical evaluation and systematic summary of the effectiveness of these interventions would help to inform policy makers in adopting interventions for expanding health insurance coverage, particularly as the World Health Organization are recommending increased coverage of social health insurance and other prepayment systems for improving the health financing system in order to achieve universal coverage (WHO 2005).

\section{O B J E C T I VES}

To assess the effectiveness of outreach strategies for expanding insurance coverage of children who are eligible for health insurance schemes.

\section{MET HO D S}

\section{Criteria for considering studies for this review}

\section{Types of studies}

The following four types of studies were included.

- Randomised controlled trials (RCTs) including clusterrandomised trials with at least two sites in each comparison group.

- Controlled clinical trials (CCTs).

- Controlled before-after (CBA) studies, provided that:

i) pre- and post-intervention periods for study and control groups are the same;

ii) the control site is appropriate (no major baseline differences between study and control groups);

iii) there are at least two intervention sites and two control sites.

- Interrupted time series (ITS) studies, provided that:

i) there is a clearly defined point in time when the intervention occurred;

ii) there are at least three data points before and three after the intervention. 


\section{Types of participants}

We included children and young people eligible for any kind of health insurance scheme but not enrolled. The age bracket of children and young people was dependent on the eligibility criteria of the health insurance scheme.

Health insurance is a health financing mechanism which involves the pooling of eligible individuals' contributions to cover all or part of the cost of certain health services for the eligible population (McIntyre 2007). Of course, some health insurance schemes subsidise or exempt contributions for certain eligible subgroups, for example social health insurance usually subsidises the premium for low-income populations (Hsiao 2007). There are three kinds of health insurance: social health insurance, community-based health insurance and private health insurance (McIntyre 2007). In this review, we do not restrict types of health insurance scheme as long as the health insurance scheme was expanding coverage of children.

\section{Types of interventions}

We included any outreach interventions that aim to improve enrolment of eligible children into existing health insurance programmes:

- Increasing awareness of schemes: television or radio advertisements, or information distribution through specific places.

- Modifying enrolment: helping families with the paperwork and the other procedures necessary to apply for health insurance; position of eligibility workers in schools or health facilities; authorising more entities to interview applicants for easier or faster application; shared eligibility with other insurance or public programmes; and other methods for improving application procedures.

- Improving management and organisation of insurance schemes: improving information systems, and training staff for better management.

If a study evaluated interventions which included both changing the design of health insurance and outreach interventions, we included it only if it differentiated the effectiveness of changing design from the effects of the outreach interventions.

\section{Types of outcome measures}

\section{Primary outcomes}

Enrolment of eligible children into health insurance programmes.

\section{Secondary outcomes}

We also extracted data on other outcomes, including measures of:

- health service utilisation;
- health status;

- attitude or satisfaction of children and their parents;

- costs of the interventions; and

- any reported adverse effects.

\section{Search methods for identification of studies}

\section{Electronic searches}

We searched the following electronic databases or websites without language or date restrictions:

- The Cochrane Effective Practice and Organisation of Care Group (EPOC) Specialised Register was searched using the Cochrane Central Register of Controlled Trials (CENTRAL) (The Cochrane Library 2009, Issue 2), 30 June 2009

- The Cochrane Central Register of Controlled Trials

(CENTRAL) (The Cochrane Library 2009, Issue 2), 2 April 2009

- PubMed (January 1951 to January 2010), 26 January 2010

- EMBASE (January 1966 to April 2009), 1 April 2009

- PsycINFO (January 1967 to April 2009), 2 April 2009

- ERIC (January 1966 to April 2009), 2 April 2009

- Public Affairs Information Service (PAIS) International (January 1972 to April 2009), 2 April 2009

- International Bibliography in Social Science (January 1951 to April 2009), 2 April 2009

- WHO library databases (WHOLIS) (January 1948 to April 2009), 6 April 2009

- Global health (January 1973 to April 2009), 3 April 2009

- World Bank -- Documents \& Reports (the earliest collecting date to April 2009), 11 April 2009

- Popline (January 1970 to April 2009), 6 April 2009

- JSTOR (January 1665 to April 2009), 4 April 2009

- EconLit (January 1969 to April 2009), 3 April 2009

- Social Science Research Network (SSRN) (January 1994 to June 2009), 4 June 2009

- IDEAS (Research Papers in Economics) (January 1927 to April 2009), 6 April 2009

- ELDIS (The earliest collecting date to April 2009), 11 April 2009

- British Library for Development Studies (BLDS) (January 1966 to April 2009), 4 April 2009

- System for Information on Grey Literature in Europe (OpenSIGLE) (the earliest collecting date to April 2009), 12 April 2009

- National Technical Information Service (NTIS) (January 1964 to April 2009), 3 April 2009

- ProQuest Dissertation \& Theses Database (January 1637 to April 2009), 5 April 2009

- ISI Proceedings (January 1990 to April 2009), 5 April 2009

- PAHO Library Catalogue (the earliest collecting date to January 2010), 6 April 2010 
- African Index Medicus (AIM) (the earliest collecting date to April 2009), 5 April 2009

- HERDIN NeON Database (the earliest collecting date to April 2009), 5 April 2009

- Thai Index Medicus (the earliest collecting date to January 2010), 6 January 2010

- Scientific and Technical Egyptian Bibliographic Database (STEB) (the earliest collecting date to April 2009), 5 April 2009

- IndMED (January 1985 to April 2009), 5 April 2009

- LILACS (the earliest collecting date to June 2009), 30 June 2009

- China National Knowledge Infrastructure (CHKD-CNKI) (January 1915 to April 2009), 6 April 2009

- Chinese Medicine Premier (Wanfang Data) (January 1988 to April 2009), 9 April 2009

- ID21 (The earliest collecting date to April 2009), 4 April 2009

The dates in bracket are the databases' time span, but for some databases or websites the earliest collecting date cannot be found. The dates after refer to the original searches performed.

The PubMed search strategy includes MESH terms and freetext terms for children, health insurance and insurance coverage. This search strategy was translated into the other electronic databases using the appropriate controlled vocabulary as applicable and free-text terms. For some simple databases and websites in which advanced or expert search methods are not available, we only used combinations of free-text terms for searching. The detailed search strategy used for all databases and websites can be found in Appendix 1.

\section{Searching other resources}

In addition, we also screened reference lists of all included papers and relevant reviews identified. We contacted authors of relevant papers to identify additional studies, including unpublished and ongoing studies. We also carried out a citation search for included studies to find more potentially relevant studies.

\section{Data collection and analysis}

\section{Selection of studies}

Two review authors (BY and LJ) scanned titles and abstracts of all articles obtained from the search and retrieved the full text of articles deemed relevant. We then independently screened the full texts of studies. We resolved any disagreements on inclusion by discussion with a third author (QM, JW or PG) or contact editor (AO).

\section{Data extraction and management}

Two authors independently carried out data extraction using a data extraction form. We extracted the following information: study design, type of intervention, site and duration of the intervention, participants, primary and secondary outcomes. We resolved any disagreements by discussion with a third author or the EPOC contact editor. We managed the data in Word.

\section{Assessment of risk of bias in included studies}

We used the EPOC criteria (EPOC 2002; Appendix 2) to assess the risk of bias for objective and subjective outcomes of all included studies. For each criterion, two authors independently described what was reported in the study, commented on the description, and judged the risk of bias. We discussed unresolved disagreements with a third author or the EPOC contact editor.

We summari sed the overall risk of bias across criteria for the outcomes of included studies. For RCTs, CCTs and CBAs we primarily considered four criteria: baseline outcome measurements; baseline characteristics measurements; incomplete outcome data addressed; and protection against contamination. If these four criteria were all scored 'yes' (met) for the outcome in a study, the summary assessment was that there was a low risk of bias; if one or more key criteria were scored 'unclear' the summary assessment was an unclear risk of bias. If one or more key criteria were scored 'no ', the summary assessment was a high risk of bias. For ITS studies, when we summari sed the overall risk of bias across these criteria, we primarily considered the following criteria: intervention independence, intervention effecting data collection and incomplete outcome data addressed.

We assessed the quality of evidence for the outcomes of included studies using the GRADE approach (GRADE 2004).

\section{Data synthesis}

We grouped study results according to the outcomes measured. For the studies we included, we present results in terms of absolute change and relative percentage change. We also calculated absolute change from baseline and the difference in absolute changes from baseline because the baseline data are available (Table 2,Table 3). We also calculated unadjusted risk ratios (RR) with $95 \%$ confidence intervals for dichotomous outcomes and mean differences (MD) with 95\% confidence intervals for continuous outcomes which were not reported by the authors. The authors of one of the included trials (Flores 2005) did not report the number of events in the intervention and control groups for the dichotomous outcomes, therefore we calculated these numbers of events using percent value and the number of participants in whom the corresponding outcome was measured. 
Table 2. Effect of awareness and application support

\begin{tabular}{|c|c|c|c|c|c|c|}
\hline Study & $\begin{array}{l}\text { Outcome } \\
\text { measure }\end{array}$ & $\begin{array}{l}\text { Absolute change } \\
\text { from baseline in } \\
\text { control group }\end{array}$ & $\begin{array}{l}\text { Absolute change } \\
\text { from baseline in } \\
\text { intervention } \\
\text { group }\end{array}$ & $\begin{array}{l}\text { Difference in ab- } \\
\text { solute change } \\
\text { from baseline }\end{array}$ & $\begin{array}{l}\text { Absolute change } \\
\text { (post) }\end{array}$ & $\begin{array}{l}\text { Relative percent- } \\
\text { age change } \\
\text { (post) }\end{array}$ \\
\hline \multirow[t]{5}{*}{ Flores 2005} & Enrolment & $57 \%$ & $96 \%$ & $39 \%$ & $39 \%$ & $68.4 \%$ \\
\hline & $\begin{array}{l}\text { Continuous en- } \\
\text { rolment }\end{array}$ & $30 \%$ & $78 \%$ & $48 \%$ & $48 \%$ & $160 \%$ \\
\hline & $\begin{array}{l}\text { Sporadic } \\
\text { enrolment }\end{array}$ & - & - & - & $-9 \%$ & $-33.3 \%$ \\
\hline & $\begin{array}{l}\text { Mean time to ob- } \\
\text { tain insurance }\end{array}$ & - & - & - & -47.3 & $-35.1 \%$ \\
\hline & $\begin{array}{l}\text { Parental satisfac- } \\
\text { tion with process } \\
\text { of enrolment }\end{array}$ & - & - & - & -1.07 & $-44.6 \%$ \\
\hline
\end{tabular}

Table 3. Effect of handing out applications in emergency department of hospitals

\begin{tabular}{|l|l|l|l|l|l|l|}
\hline Study & $\begin{array}{l}\text { Outcome } \\
\text { sure }\end{array}$ & $\begin{array}{l}\text { mea- } \\
\text { Absolute change } \\
\text { from baseline in } \\
\text { control group }\end{array}$ & $\begin{array}{l}\text { Absolute change } \\
\text { from baseline in } \\
\text { intervention } \\
\text { group }\end{array}$ & $\begin{array}{l}\text { Difference in } \\
\text { absolute change } \\
\text { from baseline }\end{array}$ & $\begin{array}{l}\text { Absolute change } \\
\text { (post) }\end{array}$ & $\begin{array}{l}\text { Rel- } \\
\text { ative percentage } \\
\text { change (post) }\end{array}$ \\
\hline Gordon 2005 & Enrolment & $28 \%$ & $42 \%$ & $14 \%$ & $14 \%$ & $50 \%$ \\
\hline
\end{tabular}

\section{RES U L T S}

\section{Description of studies}

See: Characteristics of included studies; Characteristics of excluded studies.

\section{Results of the search}

We retrieved 89 potentially relevant papers on children's health insurance coverage after screening 8761 titles and abstracts (after checking duplicates between databases). We examined the full texts of these 89 articles, and were left with 23 closely relevant papers. We then excluded 19 because they did not meet the study design inclusion criteria. One CBA study (Sieben 2000) was also excluded because inappropriate choice of control sites (there were baseline differences between participants from intervention and control sites for most characteristics, such as race/ethnicity, birthplace, language, employment status and baseline insurance coverage). One cluster-RCT study (Chen 2009) was excluded because there was only one site in each comparison group in this study. Ultimately one RCT study (Flores 2005) and one CCT (Gordon 2005) were included. The whole screening process is presented in Figure 1. 
Figure I. Screening Flow Diagram

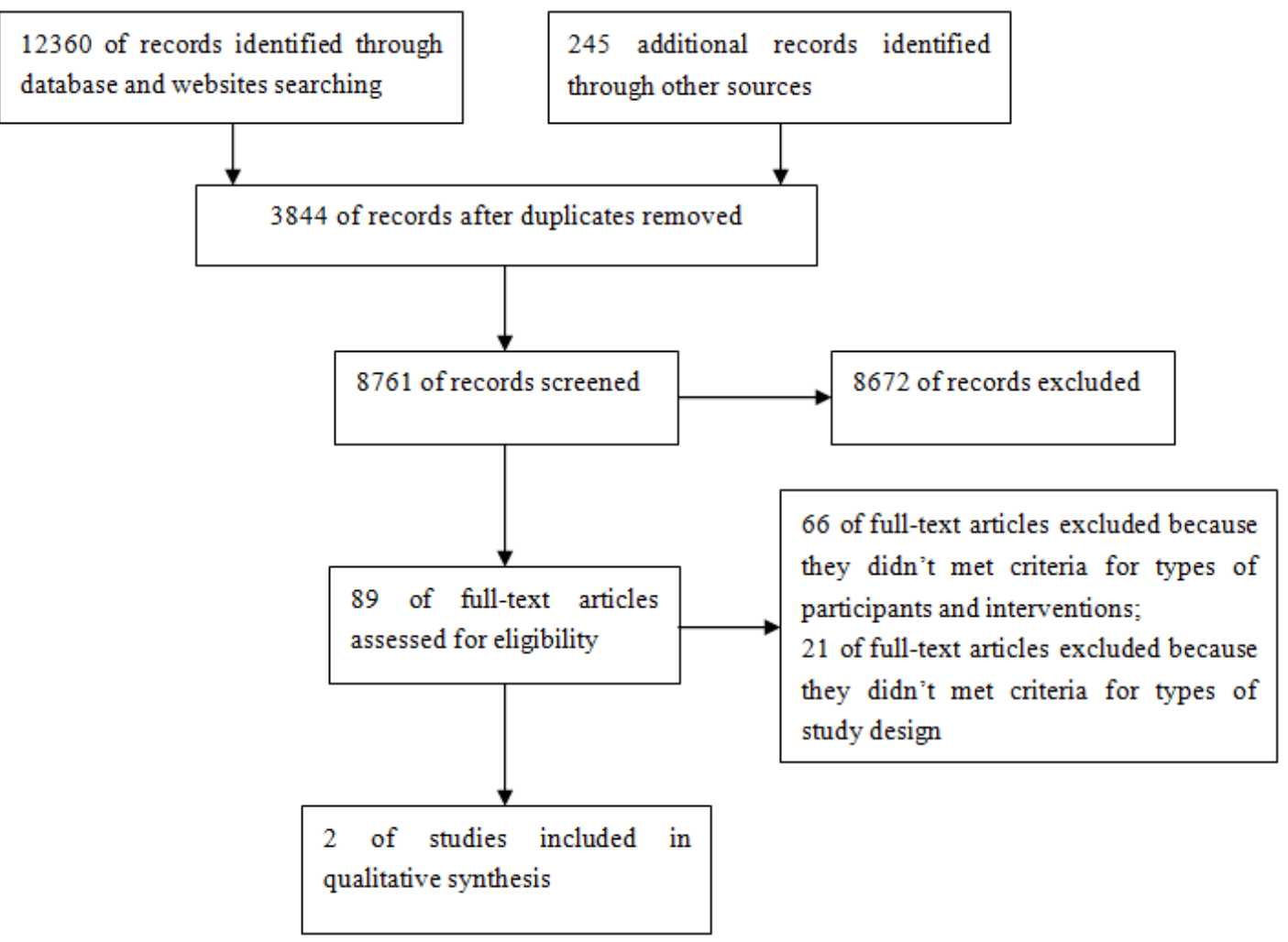

\section{Included studies}

The included RCT (Flores 2005) took place in the United States and evaluated a strategy of case management for Latino American children. From an urban community in Boston children who were not insured were recruited and randomised. The intervention group were allocated trained case managers who advised on eligibility, helped parents complete the forms, liaised with the state service to expedite decisions, helped negotiate with the state agency responsible for insurance (acting as an advocate), appealed when a child was inappropriately deemed ineligible for insurance, and followed the families up monthly for 11 months to make sure this was continued. The control group got no case manager and were just contacted monthly for 11 months. In this study "enrolment into health insurance" was the primary outcome. Some other outcome measures were also measured including continuous enrolment (keeping insured during the follow-up period), sporadic enrolment (obtaining but then losing insurance coverage during the follow-up period), mean time to obtaining insurance (the number of days from participant's study recruitment to obtaining insurance coverage) and satisfaction of parents with the process of enrolment.

Another included study is a CCT (Gordon 2005) conducted in four US cities which aimed to assess the effect of handing out applications to uninsured children in the Emergency Department (ED) of hospitals which were considered locations frequented by uninsured children. This trial recruited uninsured children younger than 18 years among patients visiting the ED into intervention and control group. The outcome measure of this trial was insurance status of children at about 90 days after ED visit.

\section{Excluded studies}

The excluded studies table (Characteristics of excluded studies) provides reasons for exclusion of 21 studies that were very close to being included but did not meet the study design requirements. Most of the excluded studies used modelling methods to analyse longitudinal data for evaluating multiple health insurance policy arrangements at the same time, which involves some possible confounding factors. Some studies just described the insurance status change after intervention without comparison with control group or pre-intervention situation. One CBA (Sieben 2000) study was 
excluded because of an inappropriate choice of control sites: baseline characteristics of participants at the control and intervention sites were very different. One cluster-RCT (Chen 2009) was excluded because there was only one site in each comparison group.

\section{Risk of bias in included studies}

The included RCT study (Flores 2005) has a low risk of bias. In this study, a computer-generated, stratified randomisation process was applied for allocation, opaque and sealed envelopes were produced for allocation concealment, and research assistants who did not participate in any aspect of the preparation of the randomisation schedules opened the envelopes in the presence of enrolled participants to inform them of their group assignment. Research assistants for the follow-up interview and outcome measurement were also blinded to group assignment at all times. Only participants were informed that some would get a case manager, whereas others would get no case manager and just be contacted monthly.
The follow-up rate was high and reported clearly.

The CCT (Gordon 2005) has an unclear risk of bias. This trial was located at four inner-city hospitals in four cities in the US. A quasirandomised allocation method was used in this trial: site staff in EDs of hospitals enrolled consecutive uninsured children in control and intervention periods; each control period was followed by a matched intervention period during which its staff handed out state-specific SCHIP applications to parents or guardians of participating children. Study periods ranged from four to 14 days; control and intervention periods were separated by about a week. The overall follow-up rate of this trial was low, with $61 \%$ of included subjects completing follow up for outcome measurement at about 90 days after visiting the ED. The characteristics of missing subjects and the follow-up rate for intervention and control groups were not reported in the article.

A methodological graph and summary can be found in Figure 2 and Figure 3.

Figure 2. Methodological quality graph: review authors' judgements about each methodological quality item presented as percentages across all included studies.

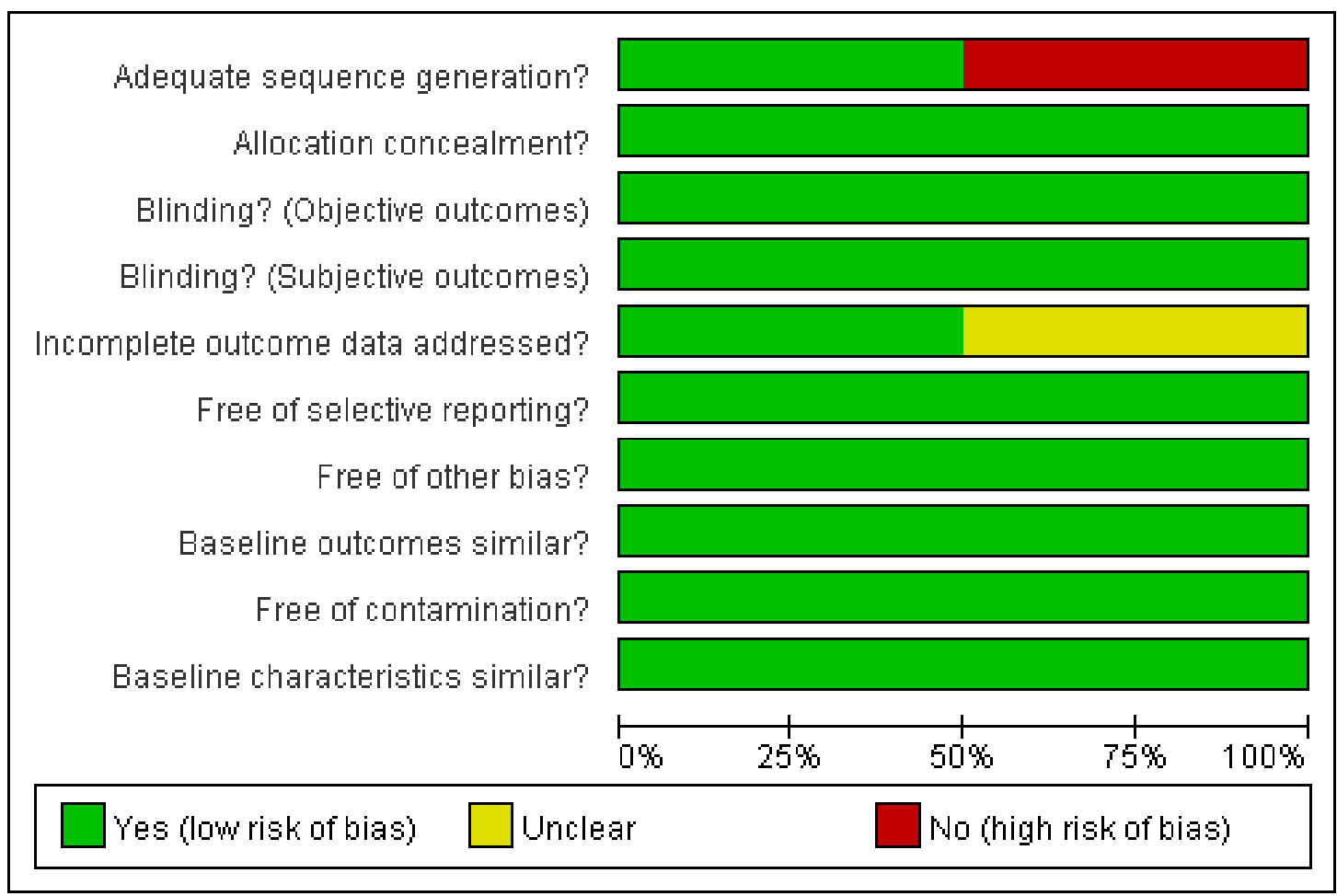


Figure 3. Methodological quality summary: review authors' judgements about each methodological quality item for each included study.

\begin{tabular}{|c|c|c|c|c|c|c|c|c|c|c|}
\hline & 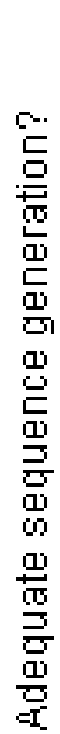 & 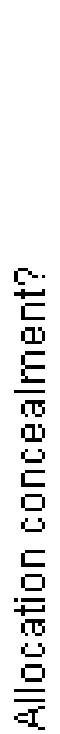 & 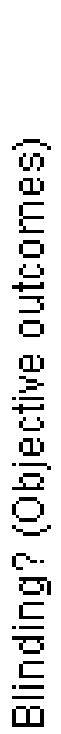 & 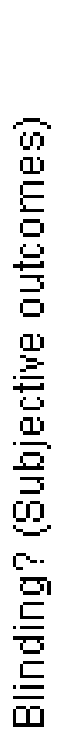 & 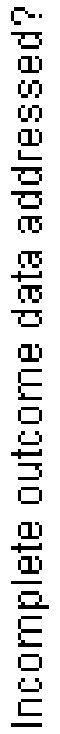 & 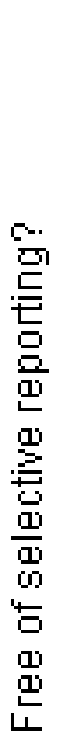 & 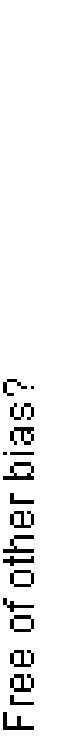 & 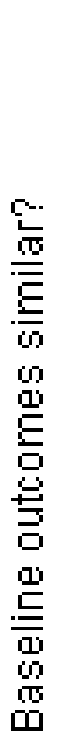 & 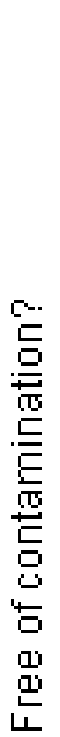 & 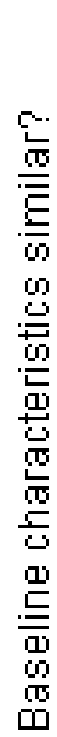 \\
\hline Flores 2005 & & & & & & & & & & \\
\hline Gordon 2005 & & & & & $?$ & & & & & \\
\hline
\end{tabular}

\section{Effects of interventions}

See: Summary of findings for the main comparison; Summary of findings 2

\section{Awareness and application support}

The RCT (Flores 2005) evaluated the effect of a community-based case manager providing insurance information and application assistance. The outcome measures in this study include enrolment into health insurance, continuously insured, sporadically insured, mean time to obtain insurance and parental satisfaction with the process of obtaining insurance.

In this trial, 275 participants were randomly assigned to intervention $(n=139)$ and control groups $(n=136)$. Beginning one month after the date of study enrolment, the participants were interviewed by telephone or visited monthly for 11 months to measure the outcomes. Four participants from the intervention group and 14 from the control group were lost prior to the first follow-up contact, so 135 (97\%) participants from the intervention group and $122(90 \%)$ from the control group were contacted at least once and measured for the outcomes "enrolment into health in- surance", "continuously insured" and "sporadically insured". The outcome "mean time to obtain insurance" was only measured for the participants obtaining insurance ( this number was derived from the percentage of obtaining participants and the total number of participants measured for obtaining insurance). The outcome "parental satisfaction with process of obtaining insurance" was only measured in the final (11th) month of follow up (97 in the intervention group, 76 in the control group).

In summary, children receiving community-based case management compared to children in control group were:

- substantially more likely to obtain health insurance coverage (96\% versus 57\%; RR 1.68 (95\% CI 1.44 to 1.96 ); $\mathrm{n}=257$ );

- more likely to be continuously insured (78\% versus $30 \%$; RR 2.59 (95\% CI 1.95 to 3.44$) ; \mathrm{n}=257)$, and less likely to be sporadically insured (18\% versus 27\%; RR 0.66 (95\% CI 0.41 to 1.05$) ; \mathrm{n}=257)$;

- quicker in obtaining insurance (mean days to obtain insurance: 87.5 versus 134.8; MD -47.30 (95\% CI -73.98 to 20.62); $\mathrm{n}=200$ (only measured on children obtaining insurance)); and 
- their parents were more satisfied with process of obtaining insurance (satisfaction score: 1.33 versus 2.40; MD -1.07 (95\% CI -1.42 to -0.72 ); $\mathrm{n}=173$ (only measured in respondents at the final follow up)).

In a multivariate analysis, the authors adjusted for potential confounders (the child's age, family income, parental citizenship, parental employment and the period of policy change in state coverage of uninsured children). It was found that the intervention group was about 1.60 times more likely than the control group to obtain insurance coverage, if the percentage of obtaining insurance coverage in control group (57\%) was used as an assumed control risk to transform the odds ratio (OR) the authors reported (OR 7.78; $95 \%$ CI 5.20 to 11.64 ) to RR (RR 1.60 ; $95 \%$ CI 1.53 to $1.65)$.

\section{Handing out applications in the emergency department of hospitals}

Gordon's CCT (Gordon 2005) assessed the effectiveness of handing out state-specific SCHIP applications in the emergency departments (ED) of hospitals. The site staff in four hospitals en- rolled uninsured children visiting the ED. There were 399 participants who met the inclusion criteria of the trial and were allocated into the intervention or control group. Two-hundred and fortytwo subjects $(61 \%)$ were followed up by both home interview (interviewing parents or guardians for children's insurance status) and state follow up (querying state databases to confirm insurance status). Nineteen participants were excluded from the final analysis because they reported receiving other insurance coverage (not Medicaid or SCHIP).

Based on 223 participants (115 intervention subjects, 108 control subjects), children receiving SCHIP applications in the ED of hospital were more likely to be insured by Medicaid or SCHIP at about 90 days after visiting the $\mathrm{ED}$ ( $42 \%$ versus $28 \%$; RR 1.50 (95\% CI 1.03 to 2.18)). By adjusting for potential confounders (age, gender, race, if having public assistance), the multivariate analysis showed that children receiving applications were about 2.1 times more likely to be insured than children in the control group, when the percentage of insured children in the control group (28\%) was used as the assumed control risk to transform the OR the authors reported (OR 3.8; $95 \%$ CI 1.7 to 8.6 ) to RR (RR 2.13; 95\% CI 1.42 to 2.75 ). 


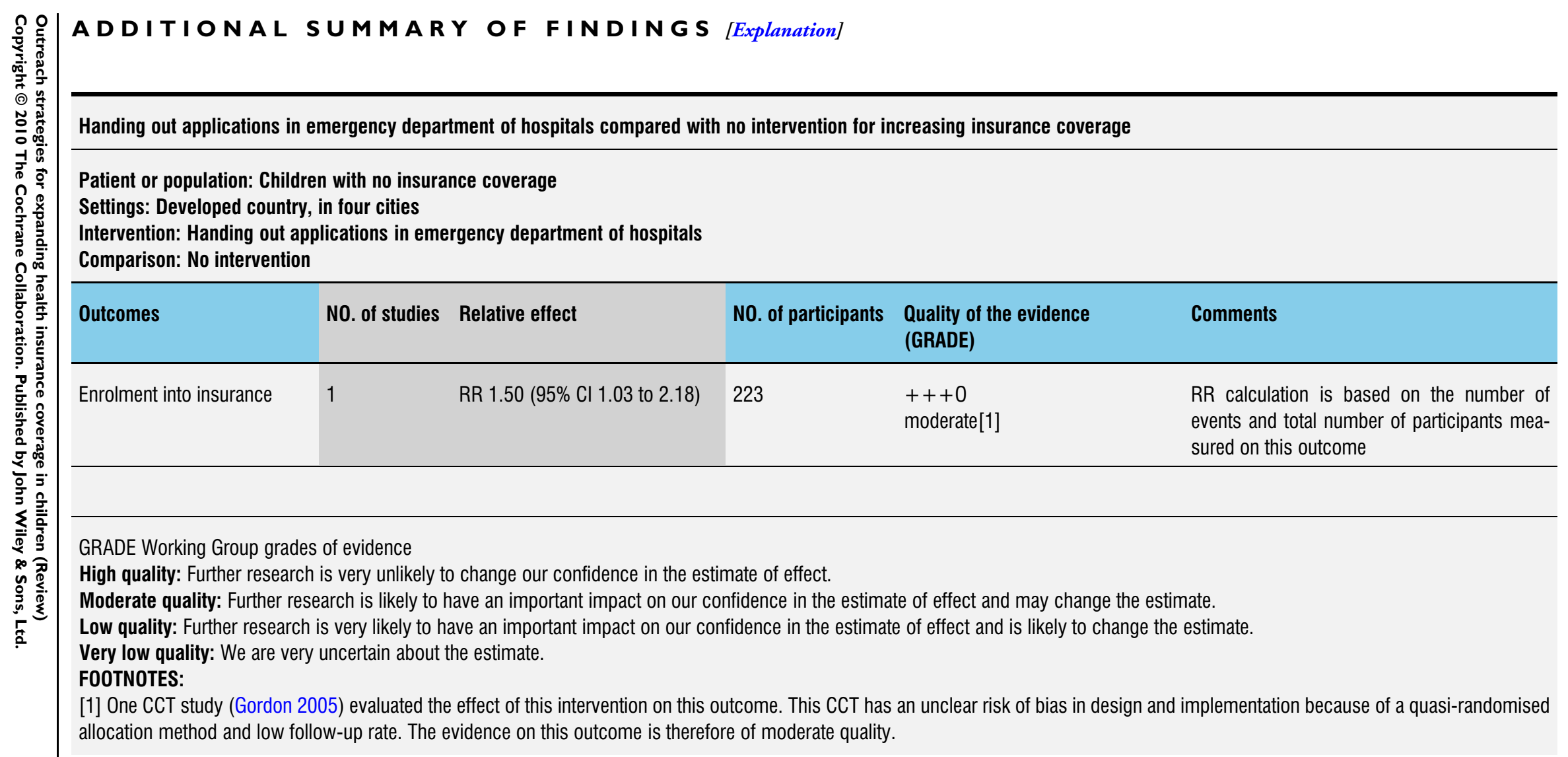




\section{DISCUSSION}

\section{Summary of main results}

This review includes two studies about outreach strategies for expanding insurance coverage of children in the US (Flores 2005; Gordon 2005). One randomised controlled trial (RCT) with a low risk of bias (Flores 2005) evaluated the effects of a community-based case manager who provided health insurance information and application assistance to Latino American children, and showed that this outreach intervention could improve the enrolment of children into health insurance schemes, keep them in health insurance and shorten the application time. Another controlled clinical trial (CCT) with an unclear risk of bias (Gordon 2005) assessed the effect of handing out insurance applications in emergency departments of hospitals, which was also shown to increase enrolment of children into health insurance schemes.

\section{Overall completeness and applicability of evidence}

The included studies are both from the US, where children's health insurance is clearly a concern of policy makers and researchers, because we identified a great many US studies about children's health insurance coverage. We did not find evidence for expanding health insurance for children in other countries. Based on our scoping review in 2008 (Meng 2010), most articles on strategies for expanding health insurance in developing countries have targeted rural and low-income populations, and only one descriptive study from Egypt introduces its health insurance programme for schoolbased children (Nandakumar 2000). However, the strategies used for rural and low-income populations are probably helpful for expanding insurance coverage of children, so confining the type of participants to children only may preclude some potentially usefulevidence.

The participants of one included study (Flores 2005) were Latino American children. The intervention in this study was specially designed in one district, and implementation of the intervention needed trained case managers who were bilingual Latina women recruited through job listings posted in the employment offices of local colleges and universities. All these factors may limit the applicability of evidence from this study to other groups of children in other districts and other countries. Another CCT targeted general uninsured children visiting four geographically diverse hospital emergency departments, so the evidence suggesting positive effects of handing out applications in emergency departments could be applicable in the US. Since the US Congress enacted the SCHIP in 1997 (Flores 2005), federal and state governments both appropriated funds for expanding Medicaid to cover more children or establishing separate health insurance programmes for more children. Medicaid and SCHIP in the US are also relatively complicated insurance schemes in terms of eligibility assessment and en- rolment procedures which involve verifying the eligibility status of families who apply for insurance for their children (Carpenter 1998). All these special contexts in the studies conducted in the US probably limit the generalisability of the evidence to other countries with different insurance schemes.

\section{Quality of the evidence}

The included RCT (Flores 2005) has a low risk of bias, but only targeted Latino American children, so the evidence based on this study is only indirectly relevant to children generally. In addition, the intervention in this trial is quite specific, with the specially trained case managers, which may not be relevant, possible or practical in other places. Overall, therefore, the evidence for all outcomes from this trial is of moderate quality (Summary of findings for the main comparison). The CCT (Gordon 2005) has an unclear risk of bias in terms of its design and implementation, and the evidence on the effects of handing out application materials in hospital emergency departments is of moderate quality (Summary of findings 2).

\section{Potential biases in the review process}

Two studies were included in this review, but quantitative synthesis of the results was not possible because different interventions were evaluated by the two studies. The data from the RCT (Flores 2005) were not fully described and we derived absolute numbers of events from the percentages and the number of participants in whom the corresponding outcome was measured: we have contacted the study authors but have not received a response.

\section{Agreements and disagreements with other studies or reviews}

There have been several literature reviews (Andrulis 1999; Dunbar 1999; Laschober 2000; Secretary of Health and Human Services 1998; Zambrana 2004) describing the studies and experiences of strategies used for expanding health insurance coverage. However, these reviews did not aim to assess the effectiveness of interventions and neither did they transparently appraise the quality of evidence.

\section{AU THORS' CONCLUSIONS}

\section{Implications for practice}

Based on the evidence included in this review, the findings suggest that intensive case management, providing insurance information, application assistance and communicating with the insurer to assist enrolment probably help increase insurance coverage of 
children in the US . Handing out insurance application materials in emergency departments in hospitals where uninsured children frequently visit also probably improves the enrolment of children into insurance schemes in the US. However, the generalisability of our findings to other counties with different health insurance schemes is unknown.

\section{Implications for research}

There is a dearth of robust studies evaluating the effects of outreach strategies for expanding health insurance coverage in children.

Many observational studies exist, however inference of an effect is not possible. Fu rthermore a cluster- randomised controlled trial and a controlled before-after study had to be excluded from this review because of serious design problems. Future research should use well- designed randomised or quasi-experimental methods to help evaluate specific strategies. Many other types of outreach strategies are used for expanding enrolment of children (such as television or radio advertisements for increasing awareness; sim- plifying application procedures; training staff for better management), so more studies evaluating other strategies or comparing the effects of different strategies would be helpful for health insurance policy makers. Lastly, future studies in this area would benefit from more research on outreach strategies used by other kinds of health insurance schemes or in other countries beyond the United States.

\section{ACKNOWLEDGEMENTS}

This review was funded by the Alliance for Health Policy and Systems Research, World Health Organization. Technical support was provided through the Effective Health Care Research Programme Consortium, supported by the Department for International Development UK. Thanks to Marit Johansen for assisting in searching several electronic databases. Particular thanks to Andy Oxman for considerable support at all stages of the review process.

\section{R E F E R E N C E S}

\section{References to studies included in this review}

Flores 2005 \{published and unpublished data\} Flores G, Abreu M, Chaisson CE, Meyers A, Sachdeva RC, Fernandez $\mathrm{H}$, et al.A randomized, controlled trial of the effectiveness of community-based case management in insuring uninsured Latino children. Pediatrics 2005;116:1433-41.

Gordon 2005 \{published and unpublished data\} Gordon JA, Emond JA, Camargo CA. The State Children's Health Insurance Program: a multicenter trial of outreach through the emergency department. American Journal of Public Health 2005;95 (2):250-3.

\section{References to studies excluded from this review}

\section{Aizer 2002 \{published and unpublished data\}}

Aizer A. Covering Kids: efforts to increase the health insurance coverage of poor children. University of California, Los Angeles 2002.

Aizer 2006 \{published and unpublished data\}

Aizer A. Public health insurance, program take-up, and child health. Working paper, National Bureau of Economic Research 2006.

Bansak 2007 \{published and unpublished data\} Bansak C, Raphael S. The effects of state policy design features on take-up and crowd-out rates for the State Children's Health Insurance Program. Journal of Policy Analysis and Management 2006;26(1):149-75.

Brown 2004 \{published and unpublished data\} Brown AM, Glazer G. Enrollment success in state children's health insurance program after free clinic referral. Journal of Pediatric Health Care 2004;18(3):145-8.
Carter 2006 \{published and unpublished data\}

Carter TM. Alabama rural partnerships: addressing the health needs of school children. United States, Alabama, The University of Alabama 2006.

\section{Chen 2009 \{published and unpublished data\}} Chen JY, Swonger S, Kominski G, Liu H, Lee JE, Diamant A. Cost-effectiveness of insuring the uninsured: the case of Korean American children. Medical Decision Making 2009;29:51-60.

Cousineau 2007 \{published and unpublished data\} Cousineau MR, Wada EO, Hogan L. Enrolling in Medicaid through the National School Lunch Program: outcome of a pilot project in California schools. Public Health Reports 2007;122(4): 452-60.

Dutton 2000 \{published and unpublished data\}

Dutton M, Katz S, Pennington A. Using community groups and student volunteers to enroll uninsured children in Medicaid and children health plus. The Commonwealth Fund 2000.

Gordon 2001 \{published and unpublished data\} Gordon JA, Dupuie TA. Child health insurance outreach through the emergency department: a pilot study. Academic Emergency Medicine 2001;8(11):1088-90.

Hill 2008 \{published and unpublished data\} Hill I, Dubay L, Kenney GM, Howell EM, Courtot B, Palmer L. Improving coverage and access for immigrant Latino children: the Los Angeles healthy kids program. Health Affair 2008;27(2):550-9.

Jacobson 2007 \{published and unpublished data\}

Jacobson M, Buchmueller TC. Can private companies contribute to public programs' outreach efforts? Evidence from California. Health Affairs 2007;26(2):538-48. 
Kincheloe 2004 \{published and unpublished data\}

Kincheloe JR. The determinants of participation in California's Medi-Cal and Healthy Families Programs by eligible children. United States, California, University of California, Los Angeles 2007.

Lee 2008 \{published and unpublished data\}

Lee HJ, Tian WH. The State Children's Health Insurance Program: participation and substitution. The Social Science Journal 2008;45 (3):382-400

Lichtenstein 2005 \{published and unpublished data\} Lichtenstein B, Sharma AK, Wheat JR. Health inequity: the plight of uninsured children in a rural Alabama county and the plan to cure it. Family and Community Health 2005;28(2):156-67.

Mahajan 2005 \{published and unpublished data\}

Mahajan P, Stanley R, Ross KW, Clark L, Sandberg K, Lichtenstein $R$. Evaluation of an emergency department-based enrollment program for uninsured children. Annals of Emergency Medicine 2005;45(3):245-50.

Meyer 2004 \{published and unpublished data\}

Meyer CL, Brun C, Yung B, Clasen C, Cauley K, Mase WA. Evaluation of social marketing efforts designed to increase enrollment in the Children's Health Insurance Program (CHIP). Journal of Nonprofit \& Public Sector Marketing 2004;12(2):87-104.

Ray 2006 \{published and unpublished data\}

Ray J, White M, Cannon P, Bowen C, O’ Rourke K. Implementing the Florida KidCare open enrollment communications campaign: a framework for mobilizing community partners to reduce the number of uninsured children. International Quarterly of Community Health Education 2006;26(4):365-77.

Sieben 2000 \{published and unpublished data\}

Sieben I, Rosenberg TJ, Bazile Y. The Role of WIC Center and Small Businesses in Enrolling Uninsured Children in Medicaid and Child Health Plus. Medical and Health Research Association of New York City, Inc. 2000.

Wada 2006 \{published and unpublished data\}

Wada EO, Cousineau MR. Assessing community-based outreach and enrollment activities and outcomes relative to need in Los Angeles County. The Center for Community Health Studies, USC Keck School of Medicine, Department of Family Medicine 2006.

Wolfe 2005 \{published and unpublished data\}

Wolfe B, Scrivner S. The devil may be in the details: how the characteristics of SCHIP programs affect take-up. Journal of Policy Analysis and Management 2005;24(3):499-522.

Ziegenfuss 2008 \{published and unpublished data\} Ziegenfuss JK. Encouraging eligible children's participation in public health insurance: the role of national awareness campaigns. United States, Minnesota, University of Minnesota 2008.

\section{Additional references}

Aizer 2006

Aizer A. Public health insurance, program take-up, and child health. Working paper, National Bureau of Economic Research 2006.

Andrulis 1999

Andrulis DP, Bauer TA, Hopkins S. Strategies to increase enrollment in children's health insurance programs: A guide to outreach, marketing and enrollment in New York and other states. A report of the New York Academy of Medicine. Journal of Urban Health 1999;76:247-79.

Arnold 1992

Arnold PJ, Schlenker TL. The impact of health care financing on childhood immunization practices. American Journal of Diseases of Children 1992;146:728-32.

Buchmueller 2007

Buchmueller TC, Jacobson M. Can private companies contribute to public outreach efforts? Evidence from California. Health Affairs 2007;26:538-48

\section{Carpenter 1998}

Carpenter BM, Kavanagh L. Outreach to children: moving from enrollment to ensuring access. Bright Futures in Managed Care Project at the National Institute for Health Care Management, the Maternal and Child Health Bureau, Health Resources and Services Administration, US Department of Health and Human Services 1998.

Carrin 2003

Carrin G. Community Based Health Insurance Schemes in developing countries: facts, problems and perspectives. World Health Organization 2003.

\section{DeNavas-Walt 2006}

DeNavas-Walt C, Proctor BD, Lee CH. Income, poverty, and health insurance coverage in the United States: 2005. Report. Washington, DC: US Government Printing Office 2005.

\section{Dunbar 1999}

Dunbar JL, Sloane HI, Mueller CD. Implementation of the State Children's Health Insurance Program: Outreach, enrollment, and provider participation in rural areas. Health Resources and Services Administration, Office of Rural health Policy 1999.

\section{EPOC 2002}

Data Collection Checklist. Cochrane Effective Practice and Organisation of Care Review Group. http:// www.epoc.cochrane.org/Files/Website\%20files/Documents/ Reviewer\%20Resources/datacollectionchecklist.pdf 2002.

\section{GRADE 2004}

The GRADE working group. Grading quality of evidence and strength of recommendations. BMJ 2004;328:1490-4.

Haley 2007

Haley J, Kenney G. Low-income uninsured children with special health care needs: why aren't they enrolled in public health insurance programs?. Pediatrics 2007;119:60-8.

Holl 1995

Holl JL, Szilagyi PG, Rodewald LE, Byrd RS, Weitzman ML. Profile of uninsured children in the United States. Archives of Pediatrics \& Adolescent Medicine 1995;149:398-406.

Hsiao 2007

Hsiao W, Shaw P. Social Health Insurance for Developing Nations. World Bank Institute: WBI Development Studies, 2007.

\section{Institute of Medicine 2002}

Institute of Medicine. Health insurance is a family matter. Report. Washington, DC: National Academies Press 2002. 


\section{Kuehl 2000}

Kuehl KS, Baffa JM, Chase GA. Insurance and education determine survival in infantile coarctation of the aorta. Journal of Health Care for the Poor and Underserved 2000;11:400-11.

\section{Langwell 2003}

Langwell K, Laschober M, Cox D, et al.American Indian and Alaska Native eligibility and enrollment in Medicaid, SCHIP and

Medicare. Report. Centers for Medicare \& Medicaid Services 2003 December.

\section{Laschober 2000}

Laschober M, Matthias S, Edder M, Quinn R. Review of the literature of evaluations of outreach for public health insurance and selected other programs. Barents Group LL, Washington, DC 2000 .

\section{Li 2001}

$\mathrm{Li} \mathrm{G}$, Davis G. Insurance status and survival outcome in pediatric trauma patients. Academic Emergency Medicine 2001;8:517A.

\section{Lu 2008}

Lu M, Zhang J, Ma J, Li B, Quan H. Child health insurance coverage: a survey among temporary and permanent residents in Shanghai. BMC Health Services Research 2008;8:238.

\section{McIntyre 2007}

McIntyre D. Learning from experience: health care financing in low- and middle-income countries. Global Forum for Health Research 2007.

\section{Meng 2010}

Meng Q, Yuan B, Jia L, Wang J, Yu B, Gao J, et al.Expanding health insurance coverage in vulnerable groups: a systematic review of options. (Accepted) 2010.

\section{Nandakumar 2000}

Nandakumar AK, Reich M, Chawla M, Berman P, Yip W. Health reform for children: the Egyptian experience with school health insurance. Health Policy 2000;50:155-70.

\section{Que 1998}

Que V. Getting healthcare to the uninsured in Georgia. Broadcast. National Public Radio “Morning Edition” 1998 August 17.

\section{Secretary of Health and Human Services 1998}

Secretary of Health and Human Services. Report to the President: Interagency Task Force on Children's Health Insurance Outreach. Washington, DC: Health Care Financing Administration June 1998.

\section{WHO 2005}

WHO. Sustainable health financing, universal coverage and social health Insurance. Ninth plenary meeting, Eighth report 2005.

\section{Zambrana 2004}

Zambrana RE, Carter-Pokras O. Improving health insurance coverage for Latino children: a review of barriers, challenges and State strategies. Journal of the National Medical Association 2004;96: 508-23.

\section{Zhang 1999}

Zhang X, Wang L, Zhu X, Wang K. Knowledge, attitude and practice survey on immunization service delivery in Guangxi and Gansu, China. Social Science \& Medicine 1999;49:1125-7.

* Indicates the major publication for the study 
CHARACTERISTICS OF STUDIES

Characteristics of included studies [ordered by study ID]

Flores 2005

\begin{tabular}{ll}
\hline Methods & RCT \\
\hline Participants & $\begin{array}{l}0 \text { to } 18 \text { years old, uninsured (for }>=3 \text { months) Latino children and their parents from } \\
2 \text { communities in the US; } 139 \text { in intervention and } 136 \text { in control } \\
\text { Recruited from May 2002 to September } 2003\end{array}$ \\
\hline
\end{tabular}

Interventions

Intervention: increasing awareness and providing application assistance (communitybased trained case managers provided information on programme eligibility, helped families complete insurance applications, acted as a family liaison with Medicaid/SCHIP, and assisted in maintaining coverage)

Control: no increasing awareness and application assistance (no case manager)

Outcomes

Health insurance coverage: determined in interview (began 1 month after date of study) with the parent and confirmed through inspection of the coverage notification letter received by the family

Continuously coverage: insured continuously through 11-months follow up

Sporadically insured: obtaining insurance but then losing coverage at any time during the 11-month follow up

Days to obtaining coverage: interval between date of the participant's recruitment and the date when they reported obtaining coverage

Parental satisfaction with the process of obtaining coverage

\begin{tabular}{|c|c|c|}
\hline Notes & \multicolumn{2}{|l|}{-} \\
\hline \multicolumn{3}{|l|}{ Risk of bias } \\
\hline Item & Authors' judgement & Description \\
\hline Adequate sequence generation? & Yes & Computer-generated, stratified randomisation process \\
\hline Allocation concealment? & Yes & $\begin{array}{l}\text { Sequentially numbered, opaque, sealed envelopes were produced } \\
\text { for each community site }\end{array}$ \\
\hline $\begin{array}{l}\text { Blinding? } \\
\text { Objective outcomes }\end{array}$ & Yes & $\begin{array}{l}\text { The primary outcomes are objective } \\
\text { Research assistants who did not participate in any aspect of } \\
\text { preparation of randomisation schedules opened the envelopes } \\
\text { in the presence of enrolled participants, to inform them of their } \\
\text { group assignment } \\
\text { Research assistants for follow-up interview and outcome mea- } \\
\text { surement were also blinded to group assignment at all times } \\
\text { Participants knew they would be in the intervention or control } \\
\text { group after allocation }\end{array}$ \\
\hline
\end{tabular}


Flores 2005 (Continued)

\begin{tabular}{|c|c|c|}
\hline $\begin{array}{l}\text { Blinding? } \\
\text { Subjective outcomes }\end{array}$ & Yes & $\begin{array}{l}\text { Parental satisfaction is subjective } \\
\text { Research assistants who did not participate in any aspect of } \\
\text { preparation of the randomisation schedules opened the en- } \\
\text { velopes in the presence of enrolled participants to inform them } \\
\text { of their group assignment } \\
\text { Research assistants for the follow-up interview and outcome } \\
\text { measurement were also blinded to group assignment at all times } \\
\text { Participants kn ew they were in the intervention or control group } \\
\text { after allocation }\end{array}$ \\
\hline $\begin{array}{l}\text { Incomplete outcome data addressed? } \\
\text { All outcomes }\end{array}$ & Yes & $\begin{array}{l}\text { Loss to follow up is clearly explained. There was a slight but } \\
\text { statistically significant difference in subjects lost to follow up } \\
\text { before follow-up interviews }(3 \% \text { of the intervention group versus } \\
9 \% \text { of the control group: } \mathrm{P}=0.04)\end{array}$ \\
\hline Free of selective reporting? & Yes & Every outcome in the Methods section is reported \\
\hline Free of other bias? & Yes & No other bias was found \\
\hline Baseline outcomes similar? & Yes & $\begin{array}{l}\text { All the participants in the intervention and control group were } \\
\text { uninsured }\end{array}$ \\
\hline Free of contamination? & Yes & $\begin{array}{l}\text { The individuals were randomised and all participants kn ew they } \\
\text { would be in the intervention or control group. For parents in } \\
\text { the intervention group, case managers provided information on } \\
\text { health insurance and assistance in obtaining insurance. It was } \\
\text { impossible for parents in the control group to get assistance from } \\
\text { case managers, however it was possible that some parents in the } \\
\text { control group could get some information on insurance and } \\
\text { its application from parents in the intervention groups if they } \\
\text { were neighbours or kn ew each other. However, this potential } \\
\text { contamination could not change the effect of the intervention. }\end{array}$ \\
\hline Baseline characteristics similar? & Yes & $\begin{array}{l}\text { No significant differences between the } 2 \text { groups in } 5 \text { character- } \\
\text { istics. Some differences in the number of parents employed full- } \\
\text { time, and recruitment time of participants between intervention } \\
\text { and control group. }\end{array}$ \\
\hline
\end{tabular}

Gordon 2005

\begin{tabular}{ll}
\hline Methods & CCT \\
\hline Participants & $\begin{array}{l}\text { Patients aged } 18 \text { years or younger visiting the Emergency Department (ED) of } 4 \text { inner- } \\
\text { city hospitals in } 4 \text { US cities during } 2001 \text { to 2002: New York City, NY; Baton Rouge, } \\
\text { La; Chicago, Ill; and Miami, Fla } \\
\text { Children's accompanying family member self-reported the children without any insur- } \\
\text { ance; no insurance status being confirmed by hospitals administrative database } \\
\text { Exclusions: children presenting to the ED in acute distress, children admitted to the }\end{array}$ \\
\hline
\end{tabular}


Gordon 2005 (Continued)

hospital or leaving the ED without treatment or against advice, children having repeat visits

399 in both intervention and control groups were recruited

\begin{tabular}{|c|c|c|}
\hline Interventions & \multicolumn{2}{|c|}{$\begin{array}{l}\text { Intervention: handing out applications in the ED (site staff in the ED handed out state- } \\
\text { specific SCHIP applications to the parent or guardian of participating children) } \\
\text { Control: no intervention }\end{array}$} \\
\hline Outcomes & \multicolumn{2}{|c|}{ Medicaid or SCHIP-insured status at 90 days } \\
\hline Notes & \multicolumn{2}{|l|}{-} \\
\hline \multicolumn{3}{|l|}{ Risk of bias } \\
\hline Item & Authors' judgement & Description \\
\hline Adequate sequence generation? & No & $\begin{array}{l}\text { Quasi-randomis ation method used: time was separated into } \\
\text { control and intervention periods ( } 4 \text { to } 14 \text { days per period) and } \\
\text { each control period was followed by a matched intervention pe- } \\
\text { riod during which visiting patients meeting the inclusion criteria } \\
\text { were recruited into intervention groups }\end{array}$ \\
\hline
\end{tabular}

Allocation concealment? Yes $\quad$ Allocation unit was study period: patients visiting in the

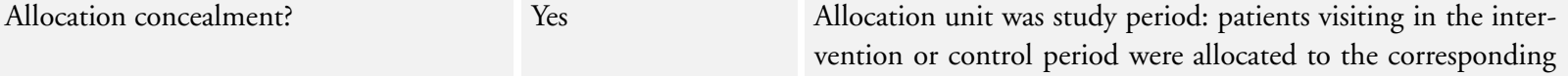
intervention or control group. Each study period ranged from 4 to 14 days; each control period was followed by a matched intervention period; control and intervention periods were separated by about a week

Blinding? $\quad$ Yes

Objective outcomes

Yes

The outcome "insurance status" is objective

No information on whether site staff measuring outcomes in follow up knew the group assignment of children; no information on whether participants knew they were in the intervention or control group after allocation. However, these could not influence the judgement of objective outcome.

\begin{tabular}{|c|c|c|}
\hline $\begin{array}{l}\text { Blinding? } \\
\text { Subjective outcomes }\end{array}$ & Yes & No subjective outcomes \\
\hline
\end{tabular}

Incomplete outcome data addressed? Unclear All outcomes
399 were recruited in both intervention and control group; 242 (61\%) completed follow up, but there was no information on the differences in follow-up rate between the intervention and control group; and no information on the characteristics of missing subjects

223 (115 in intervention group; 108 in control group) were used for analysis because $19(8 \%)$ subjects were excluded for reporting receiving other insurance (not Medicaid or SCHIP)

Free of selective reporting?
Yes 
Gordon 2005 (Continued)

\begin{tabular}{|c|c|c|}
\hline Free of other bias? & Yes & No other bias was found \\
\hline Baseline outcomes similar? & Yes & $\begin{array}{l}\text { All the participants in intervention and control group were unin- } \\
\text { sured }\end{array}$ \\
\hline Free of contamination? & Yes & $\begin{array}{l}\text { Allocation unit is study period, and control and intervention } \\
\text { periods were separated by about a week, so it was impossible for } \\
\text { subjects in the control periods to receive SCHIP applications }\end{array}$ \\
\hline Baseline characteristics similar? & Yes & $\begin{array}{l}\text { There were no significant differences in demographic character- } \\
\text { istics among control and intervention groups }\end{array}$ \\
\hline
\end{tabular}

CCT = controlled clinical trial; ED = emergency depa rtment; RCT = randomised controlled trial $;$ SCHIP = State Children Health Insurance Program

\section{Characteristics of excluded studies [ordered by study ID]}

\begin{tabular}{ll}
\hline Study & Reason for exclusion \\
\hline Aizer 2002 & $\begin{array}{l}\text { Modelling study using longitudinal data to evaluate multiple policies across multiple jurisdictions without } \\
\text { controlling for potential confounders across jurisdictions }\end{array}$ \\
\hline Aizer 2006 & Modelling study using longitudinal data before and after interventions, but not available for reanalysis \\
\hline Bansak 2007 & $\begin{array}{l}\text { Modelling study using longitudinal data to evaluate multiple policies across multiple jurisdictions without } \\
\text { controlling for potential confounders across jurisdictions }\end{array}$ \\
\hline Brown 2004 & Post- intervention description and lack of control \\
\hline Carter 2006 & $\begin{array}{l}\text { Longitudinal repeated measurements after interventions, but no measurements before interventions and no } \\
\text { control group }\end{array}$ \\
\hline Chen 2009 & Cluster-RCT, but only one site in each comparison group \\
\hline Cousineau 2007 & Post- intervention description and lack of control \\
\hline Dutton 2000 & Post- intervention survey and lack of control \\
\hline Gordon 2001 & Prospective observational study after intervention and no control group \\
\hline Aill 2008 & Analy sed longitudinal data after intervention and lack of control \\
\hline Pacosson 2007 & Postention description and lack of control \\
\hline
\end{tabular}


(Continued)

\begin{tabular}{|c|c|}
\hline Kincheloe 2004 & Cross- sectional study \\
\hline Lee 2008 & $\begin{array}{l}\text { Modelling study using longitudinal data to evaluate multiple policy characteristics without controlling for } \\
\text { possible confounding factors }\end{array}$ \\
\hline Lichtenstein 2005 & Qualitative study \\
\hline Mahajan 2005 & Post- intervention description and absence of control \\
\hline Meyer 2004 & Qualitative study \\
\hline Ray 2006 & Qualitative study \\
\hline Sieben 2000 & $\begin{array}{l}\text { Choice of control sites is not appropriate because there were significant baseline differences between partici- } \\
\text { pants from intervention and control sites for most characteristics, such as race/ethnicity, birthplace, language, } \\
\text { employment status and baseline insurance coverage }\end{array}$ \\
\hline Wada 2006 & Post- intervention description and absence of control \\
\hline Wolfe 2005 & $\begin{array}{l}\text { Analy sed pool of 2- year data to evaluate multiple insurance policy characteristics with potential confounding } \\
\text { factors }\end{array}$ \\
\hline Ziegenfuss 2008 & Modelling study using longitudinal data before and after interventions, but not available for reanalysis \\
\hline
\end{tabular}


DATA AND ANALYSES

Comparison 1. Awareness and application support versus no intervention

\begin{tabular}{lcclc} 
Outcome or subgroup title & $\begin{array}{c}\text { No. of } \\
\text { studies }\end{array}$ & $\begin{array}{c}\text { No. of } \\
\text { participants }\end{array}$ & Statistical method & Effect size \\
\hline 1 Enrolment & 1 & 257 & Risk Ratio (M-H, Fixed, 95\% CI) & $1.68[1.44,1.96]$ \\
2 Continuous enrolment & 1 & 257 & Risk Ratio (M-H, Fixed, 95\% CI) & $2.59[1.95,3.44]$ \\
$\begin{array}{l}\text { 3 Sporadic enrolment } \\
\text { 4 Mean time to obtain insurance }\end{array}$ & 1 & 257 & Risk Ratio (M-H, Fixed, 95\% CI) & $0.66[0.41,1.05]$ \\
& 1 & 200 & Mean Difference (IV, Fixed, 95\% CI) & $-47.30[-73.98,-$ \\
$\begin{array}{l}\text { Parental satisfaction with process } \\
\quad\end{array}$ & 1 & 173 & Mean Difference (IV, Fixed, 95\% CI) & $-1.07[-1.42,-0.72]$ \\
\hline
\end{tabular}

Comparison 2. Handing out applications versus no intervention

\begin{tabular}{lcccc} 
Outcome or subgroup title & $\begin{array}{c}\text { No. of } \\
\text { studies }\end{array}$ & $\begin{array}{c}\text { No. of } \\
\text { participants }\end{array}$ & Statistical method & Effect size \\
\hline 1 Enrolment & 1 & 223 & Risk Ratio (M-H, Fixed, 95\% CI) & $1.50[1.03,2.18]$ \\
\hline
\end{tabular}

\section{Analysis I.I. Comparison I Awareness and application support versus no intervention, Outcome I \\ Enrolment.}

Review: Outreach strategies for expanding health insurance coverage in children

Comparison: I Awareness and application support versus no intervention

Outcome: I Enrolment

\begin{tabular}{|c|c|c|c|c|c|}
\hline \multirow[t]{2}{*}{ Study or subgroup } & Experimental & Control & Risk Ratio & Weight & Risk Ratio \\
\hline & $\mathrm{n} / \mathrm{N}$ & $\mathrm{n} / \mathrm{N}$ & M-H,Fixed,95\% Cl & & M-H,Fixed,95\% Cl \\
\hline Flores 2005 & $130 / 135$ & $70 / 122$ & & $100.0 \%$ & $1.68[1.44,1.96]$ \\
\hline Total (95\% CI) & 135 & 122 & - & $100.0 \%$ & $1.68[1.44,1.96]$ \\
\hline
\end{tabular}

Total events: 130 (Experimental), 70 (Control)

Heterogeneity: not applicable

Test for overall effect: $Z=6.49(P<0.0000 I)$

\begin{tabular}{ccccc}
0.2 & 0.5 & 1 & 2 & 5 \\
Favours experimental & \multicolumn{3}{c}{ Favours control }
\end{tabular}


Analysis I.2. Comparison I Awareness and application support versus no intervention, Outcome 2 Continuous enrolment.

Review: Outreach strategies for expanding health insurance coverage in children

Comparison: I Awareness and application support versus no intervention

Outcome: 2 Continuous enrolment

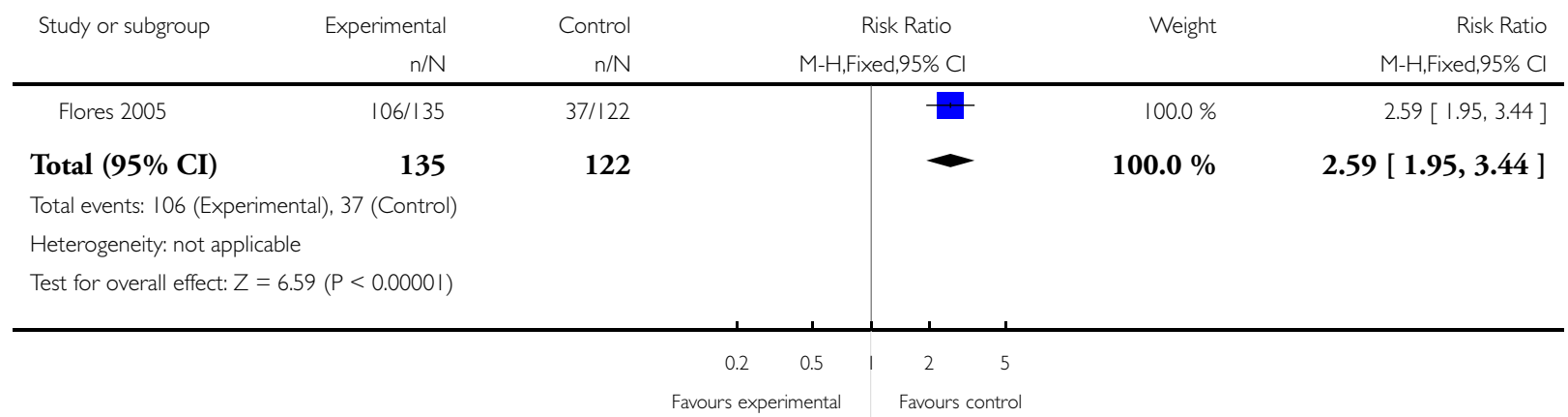

Analysis I.3. Comparison I Awareness and application support versus no intervention, Outcome 3 Sporadic enrolment.

Review: Outreach strategies for expanding health insurance coverage in children

Comparison: I Awareness and application support versus no intervention

Outcome: 3 Sporadic enrolment

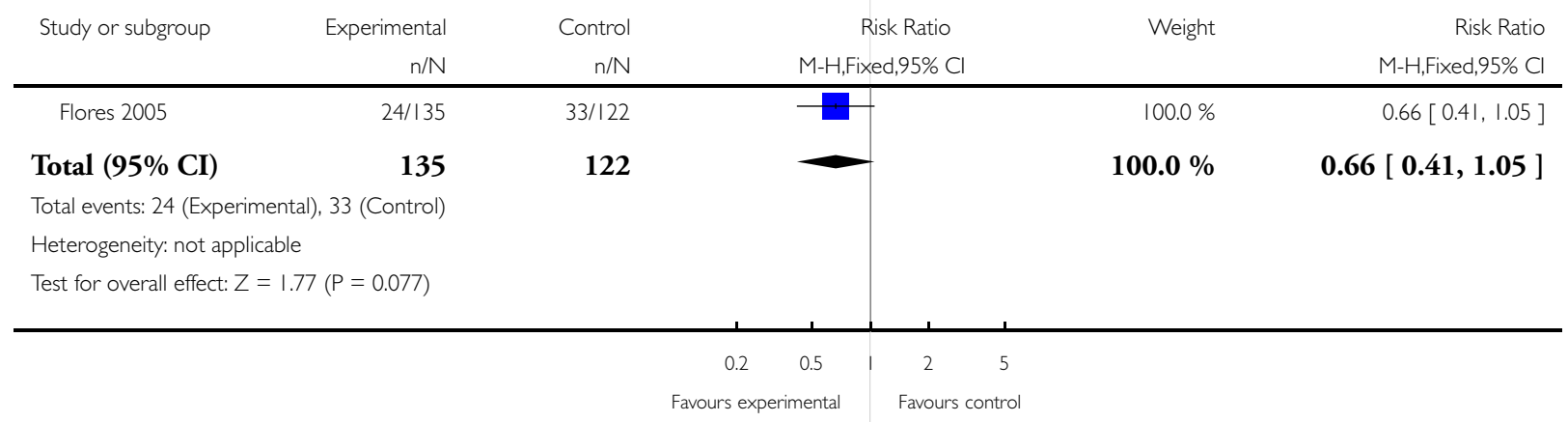


Analysis I.4. Comparison I Awareness and application support versus no intervention, Outcome 4 Mean time to obtain insurance.

Review: Outreach strategies for expanding health insurance coverage in children

Comparison: I Awareness and application support versus no intervention

Outcome: 4 Mean time to obtain insurance

\begin{tabular}{|c|c|c|c|c|c|c|c|}
\hline \multirow[t]{2}{*}{ Study or subgroup } & Experimental & \multicolumn{3}{|c|}{ Control } & Mean Difference & \multirow[t]{2}{*}{ Weight } & \multirow{2}{*}{$\begin{array}{l}\text { Mean Difference } \\
\text { IV,Fixed,95\% Cl }\end{array}$} \\
\hline & $\mathrm{N}$ & Mean(SD) & $\mathrm{N}$ & Mean(SD) & IV,Fixed,95\% Cl & & \\
\hline Flores 2005 & 130 & $87.5(68)$ & 70 & I34.8( ( 02.4$)$ & & $100.0 \%$ & $-47.30[-73.98,-20.62]$ \\
\hline
\end{tabular}

Total (95\% CI) $\quad 130$

$100.0 \% \quad-47.30[-73.98,-20.62]$

Heterogeneity: not applicable

Test for overall effect: $Z=3.47(P=0.0005 I)$

$\begin{array}{ccccc}-100 & -50 & 0 & 50 & 100 \\ \text { Favours experimental } & & \text { Favours control }\end{array}$

Analysis I.5. Comparison I Awareness and application support versus no intervention, Outcome 5 Parental satisfaction with process of enrolment.

Review: Outreach strategies for expanding health insurance coverage in children

Comparison: I Awareness and application support versus no intervention

Outcome: 5 Parental satisfaction with process of enrolment

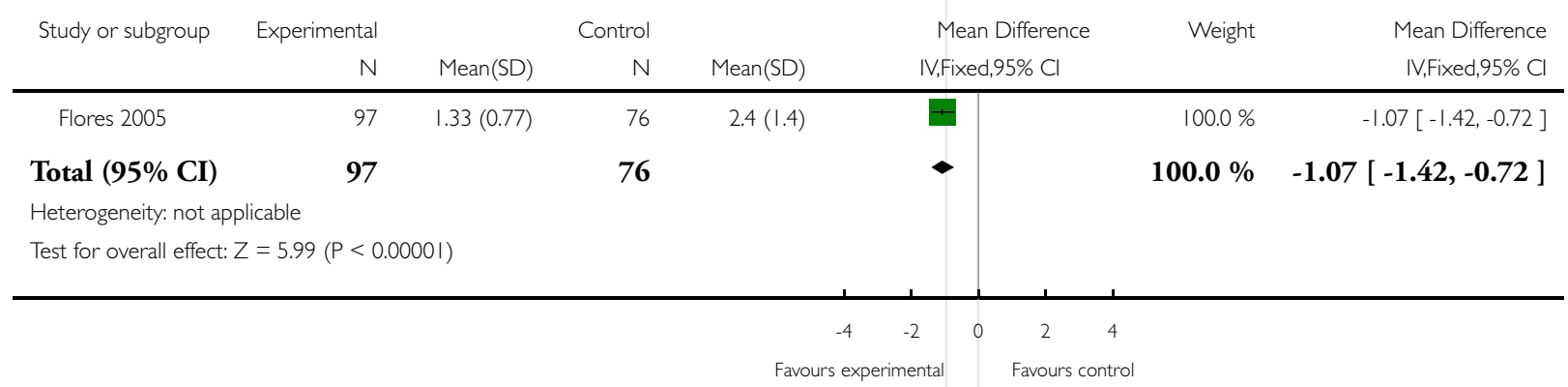


Analysis 2.I. Comparison 2 Handing out applications versus no intervention, Outcome I Enrolment.

\begin{tabular}{|c|c|c|c|c|c|}
\hline \multicolumn{6}{|c|}{ Comparison: 2 Handing out applications versus no intervention } \\
\hline Outcome: I Enrolr & & & & & \\
\hline \multirow[t]{2}{*}{ Study or subgroup } & Experimental & Control & Risk Ratio & Weight & Risk Ratio \\
\hline & $\mathrm{n} / \mathrm{N}$ & $\mathrm{n} / \mathrm{N}$ & M-H,Fixed,95\% Cl & & M-H,Fixed,95\% Cl \\
\hline Gordon 2005 & $48 / 115$ & $30 / 108$ & $\rightarrow$ & $100.0 \%$ & $1.50[1.03,2.18]$ \\
\hline Total $(95 \% \mathrm{CI})$ & 115 & 108 & - & $100.0 \%$ & $1.50[1.03,2.18]$ \\
\hline \multicolumn{6}{|c|}{ Total events: 48 (Experimental), 30 (Control) } \\
\hline \multicolumn{6}{|c|}{ Heterogeneity: not applicable } \\
\hline \multicolumn{6}{|c|}{ Test for overall effect: $Z=2.14(P=0.032)$} \\
\hline
\end{tabular}

\section{A P PENDICES}

\section{Appendix I. Search strategy}

1. EPOC Register (CENTRAL) (30 June 2009, 94 retrieved)

\#1 MeSH descriptor Child explode all trees

\#2 MeSH descriptor Infant explode all trees

\#3 MeSH descriptor Minors, this term only

\#4 MeSH descriptor Adolescent, this term only

\#5 (child or children or adolescent? or kid? or infant? or minor? or young NEXT people or young NEXT person? or teenager? or teen? ):ti,ab

\#6 (\#1 OR \#2 OR \#3 OR \#4 OR \#5)

\#7 MeSH descriptor Insurance explode all trees

\#8 MeSH descriptor Medically Uninsured, this term only

\#9 MeSH descriptor National Health Programs explode all trees

\#10 MeSH descriptor Medicare explode all trees

\#11 (health* NEAR/3 insur* or medical* NEAR/3 insur* or uninsur* or medicare or medicaid or insur* NEAR/3 program* or (cost NEXT cover*) NEAR/3 program* or (cost NEXT recover*) NEAR/3 program* or reimburs* NEAR/3 program*):ti,ab

\#12 (\#7 OR \#8 OR \#9 OR \#10 OR \#11)

\#13 (\#6 AND \#12)

$\# 14$ (sr-epoc)

\#15 (\#13 AND \#14)

2. CENTRAL (2 April 2009, 192 retrieved)

\#1 exp child/ or exp adolescent/ or exp minors/ or exp infant/ or exp students/ or (child or children or adolescent or adolescents or kid or kids or infant or infants or juvenile or juveniles or newborn or newborns or student or students or 'young people' or 'young person' or 'young persons' or teenager or teenagers or teen or teens or minor or minors or neonate or neonates or youth or youths) .ti,ab. \#2 exp financing, government/ or exp insurance/ or ('health insurance' or 'health financing' or 'medical insurance' or Medicaid or 'medical scheme' or 'medical schemes' or 'community based health organization' or 'community based health organizations' or 'community-based health organization' or 'community-based health organizations' or 'community health planning' or 'community 
health finance organization' or 'community health finance organizations' or 'community financing' or 'community self-financing' or 'mutual health organization' or 'mutual health organizations' or 'mutual health care' or 'mutual health association' or 'mutual health associations' or mutuelles or 'prepayment insurance organization' or 'prepayment insurance organizations' or 'cooperative medical scheme' or 'cooperative medical schemes' or 'cooperative medical system' or 'cooperative medical systems' or 'prepaid health care' or 'prepaid health plans' or 'prepaid health plan' or 'prepayment plan' or 'prepayment plans' or 'prepayment scheme' or 'prepayment schemes' or 'third-party payment' or 'third-party payer' or 'third party payment' or 'third party payer' or 'third-party payments' or 'thirdparty payers' or 'third party payments' or 'third party payers' or 'cost sharing' or 'risk pooling' or 'blue shield' or 'managed care programs' or 'managed care program' or 'sickness insurance') .ti,ab.

\#3 (coverage or cover or covering or covered or covers or participat* or join or joins or joining or joined or enrol* or recruit* or membership or memberships or eligib* or entitl* or 'take part in' or 'taking part in' or 'took part in' or 'taken part in' or 'takes part in' or enter or entering or entered or enters or register or registers or registration or registrations or registered or registering) .ti,ab.

\#4 1 and 2 and 3

3. PubMed (26 January 2010, 2098 retrieved)

$\# 1$ child[MH]

\#2 adolescent[$[\mathrm{MH}]$

\#3 minors[MH]

\#4 infant [MH]

\#5 students[MH]

\#6 child[TIAB] OR children[TIAB]

$\# 7$ adolescent[TIAB] OR adolescents[TIAB]

\#8 minor[TIAB] OR minors[TIAB]

\#9 infant[TIAB] OR infants[TIAB]

\#10 student[TIAB] OR students[TIAB]

\#11 kid[TIAB] OR kids[TIAB]

\#12 teenager[TIAB] OR teenagers[TIAB]

\#13 teen[TIAB] OR teens[TIAB]

$\# 14$ youth[TIAB] OR youths[TIAB]

\#15 juvenile[TIAB] OR juveniles[TIAB]

\#16 newborn[TIAB] OR newborns[TIAB]

\#17 neonate[TIAB] OR neonates[TIAB]

\#18 "young people"[TIAB]

\#19 “young person" [TIAB] OR “young persons" [TIAB]

\#20 \#1 OR \#2 OR \#3 OR \#4 OR \#5 OR \#6 OR \#7 OR \#8 OR \#9 OR \#10 OR \#11 OR \#12 OR \#13 OR \#14 OR \#15 OR \#16 OR

\#17 OR \#18 OR \#19

\#21 financing, government[MH]

\#22 insurance[MH]

\#23 "health insurance"[TIAB]

\#24 "health financing" [TIAB]

\#25 "medical insurance"[TIAB]

\#26 Medicaid[TIAB]

\#27 “medical scheme" [TIAB] OR “medical schemes"[TIAB]

\#28 “community based health organization" [TIAB] OR “community based health organizations"[TIAB]

\#29 “community-based health organization” [TIAB] OR “community-based health organizations”[TIAB]

\#30 “community health planning”[TIAB]

\#31 “community health finance organization" [TIAB] OR “community health finance organizations"[TIAB]

\#32 “community financing”[TIAB]

\#33 “community self-financing”[TIAB]

\#34 “mutual health organization”[TIAB] OR “mutual health organizations"[TIAB]

\#35 "mutual health care"[TIAB]

\#36 “mutual health association”[TIAB] OR “mutual health associations"[TIAB]

\#37 mutuelles[TIAB]

\#38 “prepayment insurance organization” [TIAB] OR “prepayment insurance organizations”[TIAB]

\#39 "cooperative medical scheme" [TIAB] OR "cooperative medical schemes"[TIAB]

Outreach strategies for expanding health insurance coverage in children (Review) 
\#40 “cooperative medical system”[TIAB] OR “cooperative medical systems”[TIAB]

\#41 "prepaid health care"[TIAB]

\#42 "prepaid health plans"[TIAB] OR "prepaid health plan"[TIAB]

\#43 "prepayment plan”[TIAB] OR “prepayment plans”[TIAB]

\#44 “prepayment scheme”[TIAB] OR “prepayment schemes”[TIAB]

\#45 “third-party payment”[TIAB] OR “third-party payer" [TIAB] OR “third party payment”[TIAB] OR "third party payer" [TIAB]

\#46 “third-party payments" [TIAB] OR “third-party payers" [TIAB] OR "third party payments"[TIAB] OR "third party payers" [TIAB]

\#47 “cost sharing”[TIAB] OR "risk pooling”[TIAB]

\#48 "blue shield"[TIAB]

\#49 “managed care programs" [TIAB] OR “managed care program”[TIAB]

\#50 "sickness insurance" [TIAB]

\#51 \#21 OR \#22 OR \#23 OR \#24 OR \#25 OR\#26 OR \#27 OR\#28 OR \#29 OR \#30 OR \#31 OR \#32 OR \#33 OR \#34 OR \#35 OR \#36 OR \#37 OR \#38 OR \#39 OR \#40 OR \#41 OR \#42 OR \#43 OR \#44 OR \#45 OR \#46 OR \#47 OR \#48 OR \#49 OR \#50 \#52 coverage[TIAB] OR cover[TIAB] OR covering[TIAB] OR covered[TIAB] OR covers[TIAB]

\#53 participat*[TIAB]

\#54 join[TIAB] OR joins[TIAB] OR joining[TIAB] OR joined[TIAB]

$\# 55$ enrol*[TIAB] $^{*}$

\#56 recruit*[TIAB]

\#57 membership[TIAB] OR memberships[TIAB]

\#58 eligib*[TIAB]

\#59 entitl*[TIAB]

\#60 “take part in”[TIAB] OR “taking part in”[TIAB] OR “took part in”[TIAB] OR “taken part in”[TIAB] OR “takes part in”[TIAB] $\# 61$ enter[TIAB] OR entering[TIAB] OR entered[TIAB] OR enters[TIAB]

\#62 register[TIAB] OR registers[TIAB] OR registration[TIAB] OR registrations[TIAB] OR registered[TIAB] OR registering[TIAB] \#63 \#52 OR \#53 OR \#54 OR \#55 OR \#56 OR \#57 OR \#58 OR \#59 OR \#60 OR \#61 OR \#62

\#64 \#20 AND \#51 AND \#63

\#65 randomized controlled trial[PT] OR random*[TIAB] OR intervention*[TIAB] OR control[TIAB] OR controll[TIAB] OR controls[TIAB] OR controlls[TIAB] OR controles[TIAB] OR controlles[TIAB] OR controled[TIAB] OR controlled[TIAB] OR controld[TIAB] OR controlld[TIAB] OR evaluat*[TIAB]

\#66 “Animals”[MH] NOT (“Animals”[MH] AND “Humans”[MH])

\#67 \#65 NOT \#66

\#68 \#64 AND \#67

4. EMBASE (1 April 2009, 1877 retrieved)

\#1 exp child/ OR exp adolescent/ OR exp newborn/ OR exp student/ OR (child OR children OR adolescent OR adolescents OR kid OR kids OR infant OR infants OR juvenile OR juveniles OR newborn OR newborns OR student OR students OR 'young people' OR 'young person' OR 'young persons' OR teenager OR teenagers OR teen OR teens OR minor OR minors OR neonate OR neonates OR youth OR youths).ti,ab.

\#2 exp insurance/ OR ('health insurance' OR 'health financing' OR 'medical insurance' OR Medicaid OR 'medical scheme' OR 'medical schemes' OR 'community based health organization' OR 'community based health organizations' OR 'community-based health organization' OR 'community-based health organizations' OR 'community health planning' OR 'community health finance organization' OR 'community health finance organizations' OR 'community financing' OR 'community self-financing' OR 'mutual health organization' OR 'mutual health organizations' OR 'mutual health care' OR 'mutual health association' OR 'mutual health associations' OR mutuelles OR 'prepayment insurance organization' OR 'prepayment insurance organizations' OR 'cooperative medical scheme' OR 'cooperative medical schemes' OR 'cooperative medical system' OR 'cooperative medical systems' OR 'prepaid health care' OR 'prepaid health plans' OR 'prepaid health plan' OR 'prepayment plan' OR 'prepayment plans' OR 'prepayment scheme' OR 'prepayment schemes' OR 'third-party payment' OR 'third-party payer' OR 'third party payment' OR 'third party payer' OR 'thirdparty payments' OR 'third-party payers' OR 'third party payments' OR 'third party payers' OR 'cost sharing' OR 'risk pooling' OR 'blue shield' OR 'managed care programs' OR 'managed care program' OR 'sickness insurance').ti,ab.

\#3 (coverage OR cover OR covering OR covered OR covers OR "participat"” OR join OR joins OR joining OR joined OR "enrol*” OR “recruit*” OR membership OR memberships OR “eligib*” OR “entitl”” OR 'take part in' OR 'taking part in' OR 'took part in' OR 'taken part in' OR 'takes part in' OR enter OR entering OR entered OR enters OR register OR registers OR registration OR registrations $\mathrm{OR}$ registered OR registering).ti,ab.

Outreach strategies for expanding health insurance coverage in children (Review) 
\#4 Randomized controlled trial/ OR (random\$ OR experiment\$ OR (time adj series) OR pre test OR pretest OR post test OR posttest OR impact OR intervention\$ OR chang\$ OR evaluat\$ OR effect? OR compar\$ OR control\$).tw.

\#5 \#4 NOT Nonhuman/

\#6 \#1AND \#2 AND \#3 AND \#5

5. PsycINFO (2 April 2009, 481 retrieved)

1 (child or children or adolescent or adolescents or kid or kids or infant or infants or juvenile or juveniles or newborn or newborns or student or students or "young people" or "young person" or "young persons" or teenager or teenagers or teen or teens or minor or minors or neonate or neonates or youth or youths):Keywords OR (child or children or adolescent or adolescents or kid or kids or infant or infants or juvenile or juveniles or newborn or newborns or student or students or "young people" or "young person" or "young persons" or teenager or teenagers or teen or teens or minor or minors or neonate or neonates or youth or youths):Abstract

2 ("health insurance" or "health financing" or "medical insurance" or Medicaid or "medical scheme" or "medical schemes" or "community based health organization" or "community based health organizations" or "community-based health organization" or "community-based health organizations" or "community health planning" or "community health finance organization" or "community health finance organizations" or "community financing" or "community self-financing" or "mutual health organization" or "mutual health organizations" or "mutual health care" or "mutual health association" or "mutual health associations" or mutuelles or "prepayment insurance organization" or "prepayment insurance organizations" or "cooperative medical scheme" or "cooperative medical schemes" or "cooperative medical system" or "cooperative medical systems" or "prepaid health care" or "prepaid health plans" or "prepaid health plan" or "prepayment plan" or "prepayment plans" or "prepayment scheme" or "prepayment schemes" or "third-party payment" or "third-party payer" or "third party payment" or "third party payer" or "third-party payments" or "third-party payers" or "third party payments" or "third party payers" or "cost sharing" or "risk pooling" or "blue shield" or "managed care programs" or "managed care program" or "sickness insurance"):Keywords OR ("health insurance" or "health financing" or "medical insurance" or Medicaid or "medical scheme" or "medical schemes" or "community based health organization" or "community based health organizations" or "community-based health organization" or "community-based health organizations" or "community health planning" or "community health finance organization" or "community health finance organizations" or "community financing" or "community self-financing" or "mutual health organization" or "mutual health organizations" or "mutual health care" or "mutual health association" or "mutual health associations" or mutuelles or "prepayment insurance organization" or "prepayment insurance organizations" or "cooperative medical scheme" or "cooperative medical schemes" or "cooperative medical system" or "cooperative medical systems" or "prepaid health care" or "prepaid health plans" or "prepaid health plan" or "prepayment plan" or "prepayment plans" or "prepayment scheme" or "prepayment schemes" or "third-party payment" or "third-party payer" or "third party payment" or "third party payer" or "third-party payments" or "third-party payers" or "third party payments" or "third party payers" or "cost sharing" or "risk pooling" or "blue shield" or "managed care programs" or "managed care program" or "sickness insurance"):Abstract

3 (coverage or cover or covering or covered or covers or participat* or join or joins or joining or joined or enrol* or recruit* or membership or memberships or eligib* or entitl* or "take part in" or "taking part in" or "took part in" or "taken part in" or "takes part in" or enter or entering or entered or enters or register or registers or registration or registrations or registered or registering):Keywords OR (coverage or cover or covering or covered or covers or participat* or join or joins or joining or joined or enrol* or recruit* or membership or memberships or eligib* or entitl* or "take part in" or "taking part in" or "took part in" or "taken part in" or "takes part in" or enter or entering or entered or enters or register or registers or registration or registrations or registered or registering):Abstract

41 and 2 and 3

6. ERIC (2 April 2009, 887 retrieved)

$1 \mathrm{KW}=$ (child or children or adolescent or adolescents or kid or kids or infant or infants or juvenile or juveniles or newborn or newborns or student or students or young people or young person or young persons or teenager or teenagers or teen or teens or minor or minors or neonate or neonates or youth or youths)

$2 \mathrm{KW}=$ (health insurance or health financing or medical insurance or Medicaid or medical scheme or medical schemes or community based health organization or community based health organizations or community-based health organization or communitybased health organizations or community health planning or community health finance organization or community health finance organizations or community financing or community self-financing or mutual health organization or mutual health organizations or mutual health care or mutual health association or mutual health associations or mutuelles or prepayment insurance organization or prepayment insurance organizations or cooperative medical scheme or cooperative medical schemes or cooperative medical system or cooperative medical systems or prepaid health care or prepaid health plans or prepaid health plan or prepayment plan or prepayment plans or prepayment scheme or prepayment schemes or third-party payment or third-party payer or third party payment or third party payer or third-party payments or third-party payers or third party payments or third party payers or cost sharing or risk pooling or blue shield or managed care programs or managed care program or sickness insurance)

Outreach strategies for expanding health insurance coverage in children (Review) 
$3 \mathrm{KW}=$ (coverage or cover or covering or covered or covers or participat* or join or joins or joining or joined or enrol* or recruit* or membership or memberships or eligib* or entitl* or take part in or taking part in or took part in or taken part in or takes part in or enter or entering or entered or enters or register or registers or registration or registrations or registered or registering)

41 and 2 and 3

7. PAIS (2 April 2009, 373 retrieved)

\#1 KW=child OR children OR adolescent OR adolescents OR minor OR minors OR infant OR infants OR student OR students OR kid OR kids OR teenager OR teenagers OR teen OR teens OR youth OR youths OR juvenile OR juveniles? OR newborn OR newborns OR neonate OR neonates OR young people OR young person OR young persons

\#2 KW=health insurance OR health financing OR medical insurance OR Medicaid OR medical scheme OR medical schemes OR community based health organization OR community based health organizations OR community-based health organization OR community-based health organizations OR community health planning OR community health finance organization OR community health finance organizations OR community financing OR community self-financing OR mutual health organization OR mutual health organizations OR mutual health care OR mutual health association OR mutual health associations OR mutuelles OR prepayment insurance organization OR prepayment insurance organizations OR cooperative medical scheme OR cooperative medical schemes OR cooperative medical system OR cooperative medical systems OR prepaid health care OR prepaid health plan OR prepaid health plans OR prepayment plan OR prepayment plans OR prepayment scheme OR prepayment schemes OR third-party payment OR thirdparty payer OR third party payment OR third party payer OR third-party payments OR third-party payers OR third party payments OR third party payers OR cost sharing OR risk pooling OR blue shield OR managed care program OR managed care programs OR sickness insurance

\#3 KW=coverage OR cover OR covering OR covered OR covers OR participat* OR join OR joins OR joining OR joined OR enrol* OR recruit* OR membership OR memberships OR eligib* OR entitl* OR take part in OR taking part in OR took part in OR taken part in OR takes part in OR enter OR entering OR entered OR enters OR register OR registers OR registration OR registrations OR registered $\mathrm{OR}$ registering

\#4 \#1 AND \#2 AND \#3

8. IBSS (2 April 2009, 82 retrieved)

\#1 exp adolescents/ OR exp students/ OR exp children/ OR exp youth/ OR exp adolescence/ OR (child OR children OR adolescent OR adolescents OR kid OR kids OR infant OR infants OR juvenile OR juveniles OR newborn OR newborns OR student OR students OR young people OR young person OR young persons OR teenager OR teenagers OR teen OR teens OR minor OR minors OR neonate OR neonates OR youth OR youths).ab,ti.

\#2 exp insurance/ OR (health insurance OR health financing OR medical insurance OR Medicaid OR medical scheme OR medical schemes OR community based health organization OR community based health organizations OR community-based health organization OR community-based health organizations OR community health planning OR community health finance organization OR community health finance organizations OR community financing OR community self-financing OR mutual health organization OR mutual health organizations OR mutual health care OR mutual health association OR mutual health associations OR mutuelles OR prepayment insurance Organization OR prepayment insurance organizations OR cooperative medical scheme OR cooperative medical schemes OR cooperative medical system OR cooperative medical systems OR prepaid health care OR prepaid health plans OR prepaid health plan OR prepayment plan OR prepayment plans OR prepayment scheme OR prepayment schemes OR third-party payment OR third-party payer OR third party payment OR third party payer OR third-party payments OR third-party payers OR third party payments OR third party payers OR cost sharing OR risk pooling OR blue shield OR managed care programs OR managed care program OR sickness insurance) .ab,ti.

\#3 (coverage OR cover OR covering OR covered OR covers OR “participat*” OR join OR joins OR joining OR joined OR "enrol*” OR “recruit*” OR membership OR memberships OR "eligib*” OR “entitl*” OR take part in OR taking part in OR took part in OR taken part in OR takes part in OR enter OR entering OR entered OR enters OR register OR registers OR registration OR registered OR registering ).ab,ti.

\#4 \#1 AND \#2 AND \#3

9. WHOLIS (6 April 2009, 15 retrieved)

1 subject "child OR adolescent OR minors OR infant OR students" OR title "child OR children OR adolescent OR adolescents OR minor OR minors OR infant OR infants OR student OR students OR kid OR kids OR teenager OR teenagers OR teen OR teens OR youth OR youths OR juvenile OR juveniles OR newborn OR newborns OR neonate OR neonates OR 'young people' OR 'young person' OR 'young persons"' OR words or phrase "child OR children OR adolescent OR adolescents OR minor OR minors OR infant OR infants OR student OR students OR kid OR kids OR teenager OR teenagers OR teen OR teens OR youth OR youths OR juvenile OR juveniles OR newborn OR newborns OR neonate OR neonates OR 'young people' OR 'young person' OR 'young persons"'

Outreach strategies for expanding health insurance coverage in children (Review)

Copyright $(2010$ The Cochrane Collaboration. Published by John Wiley \& Sons, Ltd. 
2 subject “financing, government OR insurance" OR title "'health insurance' OR 'health financing' OR 'medical insurance' OR Medicaid OR 'medical scheme' OR 'medical schemes' OR ' community based health organization' OR 'community based health organizations' OR 'community-based health organization' OR 'community-based health organizations' OR 'community health planning' OR 'community health finance organization' OR 'community health finance organizations' OR 'community financing' OR 'community self-financing' OR 'mutual health organization' OR 'mutual health organizations' OR 'mutual health care' OR 'mutual health association' OR 'mutual health associations' OR mutuelles OR 'prepayment insurance organization' OR 'prepayment insurance organizations' OR 'cooperative medical scheme' OR 'cooperative medical schemes' OR 'cooperative medical system' OR 'cooperative medical systems' OR 'prepaid health care' OR 'prepaid health plan' OR 'prepaid health plans' OR 'prepayment plan' OR 'prepayment plans' OR 'prepayment scheme' OR 'prepayment schemes' OR 'third-party payment' OR 'third-party payer' OR 'third party payment' OR 'third party payer' OR 'third-party payments' OR 'third-party payers' OR 'third party payments' OR 'third party payers' OR 'cost sharing' OR 'risk pooling' OR 'blue shield' OR 'managed care program' OR 'managed care programs' OR 'sickness insurance"' OR words or phrase "'health insurance' OR 'health financing'OR 'medical insurance' OR Medicaid OR 'medical scheme' OR 'medical schemes' OR 'community based health organization' OR 'community based health organizations' OR 'community-based health organization' OR 'communitybased health organizations' OR 'community health planning' OR 'community health finance organization' OR 'community health finance organizations' OR 'community financing' OR 'community self-financing' OR 'mutual health organization' OR 'mutual health organizations' OR 'mutual health care' OR 'mutual health association' OR 'mutual health associations' OR mutuelles OR 'prepayment insurance organization' OR 'prepayment insurance organizations' OR 'cooperative medical scheme' OR 'cooperative medical schemes' OR 'cooperative medical system' OR 'cooperative medical systems' OR 'prepaid health care' OR 'prepaid health plan' OR 'prepaid health plans' OR 'prepayment plan' OR 'prepayment plans' OR 'prepayment scheme' OR 'prepayment schemes' OR 'third-party payment' OR 'third-party payer' OR 'third party payment' OR 'third party payer' OR 'third-party payments' OR 'third-party payers' OR 'third party payments' OR 'third party payers' OR 'cost sharing' OR 'risk pooling' OR 'blue shield' OR 'managed care program' OR 'managed care programs' OR 'sickness insurance' "

31 AND 2

10. Global health (3 April 2009, 472 retrieved)

1 exp children/ OR exp adolescents/ OR exp infants/ OR exp students/ OR exp youth/ OR (child or children or adolescent or adolescents or minor or minors or infant or infants or student or students or kid or kids or teenager or teenagers or teen or teens or youth or youths or juvenile or juveniles or newborn or newborns or neonate or neonates or young people or young person or young persons).ti,ab.

2 exp insurance/ OR exp health insurance/ OR exp third party payments/ OR (health insurance OR health financing OR medical insurance OR Medicaid OR medical scheme OR medical schemes OR community based health organization OR community based health organizations OR community-based health organization OR community-based health organizations OR community health planning OR community health finance organization OR community health finance organizations OR community financing OR community self-financing OR mutual health organization OR mutual health organizations OR mutual health care OR mutual health association OR mutual health associations OR mutuelles OR prepayment insurance organization OR prepayment insurance organizations OR cooperative medical scheme OR cooperative medical schemes OR cooperative medical system OR cooperative medical systems OR prepaid health care OR prepaid health plan OR prepaid health plans OR prepayment plan OR prepayment plans OR prepayment scheme OR prepayment schemes OR third-party payment OR third-party payer OR third party payment OR third party payer OR third-party payments OR third-party payers OR third party payments OR third party payers OR cost sharing OR risk pooling OR blue shield OR managed care program OR managed care programs OR sickness insurance).ti,ab.

3 (coverage OR cover OR covering OR covered OR covers OR participat* OR join OR joins OR joining OR joined OR enrol* OR recruit* OR membership OR memberships OR eligib* OR entitl* OR take part in OR taking part in OR took part in OR taken part in OR takes part in OR enter OR entering OR entered OR enters OR register OR registers OR registration OR registrations OR registered OR registering).ti,ab.

41 AND 2 AND 3

11. World Bank - Documents \& Reports (11 April 2009, 22 retrieved)

With the exact phrase: health insurance; health financing; medical insurance; Medicaid ; medical scheme ; community based health organization; community-based health organization; community health planning; community health finance organization; community financing; community self-financing; mutual health organization; mutual health care; mutual health association; mutuelles; prepayment insurance organization; cooperative medical scheme; cooperative medical system; prepaid health care; prepaid health plan; prepayment plan; prepayment scheme; third-party payment; third-party payer; third party payment ; third party payer ; cost sharing ; risk pooling; blue shield; managed care program ; sickness insurance

12. Popline (6 April 2009, 652 retrieved)

Outreach strategies for expanding health insurance coverage in children (Review) 
SUBJECT: (child / children / adolescent / adolescents / kid / kids / infant / infants / juvenile / juveniles / newborn / newborns / student / students / young people / young person / young persons / teenager / teenagers / teen / teens / minor / minors / neonate / neonates / youth / youths) \& (health insurance / health financing / medical insurance / Medicaid /? medical scheme / medical schemes / community based health organization / community based health organizations / community-based health organization / community-based health organizations / community health planning / community health finance organization / community health finance organizations / community financing / community self-financing / mutual health organization / mutual health organizations / mutual health care / mutual health association / mutual health associations / mutuelles / prepayment insurance organization / prepayment insurance organizations / cooperative medical scheme / cooperative medical schemes / cooperative medical system / cooperative medical systems / prepaid health care / prepaid health plans / prepaid health plan / prepayment plan / prepayment plans / prepayment scheme / prepayment schemes / third-party payment / third-party payer / third party payment / third party payer / third-party payments / thirdparty payers / third party payments / third party payers / cost sharing / risk pooling / blue shield / managed care programs / managed care program /? sickness insurance) \& (coverage / cover / covering / covered / covers / participat* / join / joins / joining / joined / enrol* / recruit* / membership / memberships / eligib* / entitl* / take part in /? taking part in / took part in / taken part in / takes part in / enter / entering / entered / enters / register / registers / registration / registrations / registered / registering)

13. JSTOR (4 April 2009, 207 retrieved)

1 (ti:(child OR children OR adolescent OR adolescents OR minor OR minors OR infant OR infants OR student OR students OR kid OR kids OR teenager OR teenagers OR teen OR teens OR youth OR youths OR juvenile OR juveniles OR newborn OR newborns OR neonate OR neonates OR "young people" OR “young person" OR “young persons") OR ab:(child OR children OR adolescent OR adolescents OR minor OR minors OR infant OR infants OR student OR students OR kid OR kids OR teenager OR teenagers OR teen OR teens OR youth OR youths OR juvenile OR juveniles OR newborn OR newborns OR neonate OR neonates OR "young people" OR “young person” OR “young persons"))

2 (ti:(insurance OR "health insurance" OR "health financing” OR "medical insurance" OR Medicaid OR "medical scheme" OR "medical schemes" OR "community based health organization" OR "community based health organizations" OR "community-based health organization" OR "community-based health organizations" OR "community health planning" OR "community health finance organization" OR "community health finance organizations" OR "community financing” OR "community self-financing” OR "mutual health organization" OR "mutual health organizations" OR "mutual health care" OR "mutual health association" OR "mutual health associations" OR mutuelles OR "prepayment insurance organization” OR "prepayment insurance organizations" OR "cooperative medical scheme" OR "cooperative medical schemes" OR "cooperative medical system" OR "cooperative medical systems" OR "prepaid health care” OR "prepaid health plan” OR "prepaid health plans” OR "prepayment plan” OR "prepayment plans” OR "prepayment scheme" OR "prepayment schemes" OR "third-party payment” OR "third-party payer" OR "third party payment" OR "third party payer" OR "third-party payments" OR "third-party payers" OR "third party payments" OR "third party payers" OR "cost sharing" OR "risk pooling” OR "blue shield" OR "managed care program” OR "managed care programs" OR "sickness insurance") OR ab:( insurance OR "health insurance” OR "health financing” OR "medical insurance” OR Medicaid OR "medical scheme” OR "medical schemes" OR "community based health organization" OR "community based health organizations" OR "community-based health organization" OR "community-based health organizations" OR "community health planning" OR "community health finance organization" OR "community health finance organizations" OR "community financing” OR "community self-financing” OR "mutual health organization" OR "mutual health organizations" OR "mutual health care" OR "mutual health association" OR "mutual health associations" OR mutuelles OR "prepayment insurance organization" OR "prepayment insurance organizations" OR "cooperative medical scheme" OR "cooperative medical schemes" OR "cooperative medical system" OR "cooperative medical systems" OR "prepaid health care" OR "prepaid health plan” OR "prepaid health plans” OR "prepayment plan” OR "prepayment plans" OR "prepayment scheme” OR "prepayment schemes” OR "third-party payment” OR "third-party payer” OR "third party payment” OR "third party payer" OR "third-party payments" OR "third-party payers" OR "third party payments" OR "third party payers” OR "cost sharing” OR "risk pooling” OR "blue shield" OR "managed care program" OR "managed care programs" OR "sickness insurance”))

3 (ti: ( coverage OR cover OR covering OR covered OR covers OR participat* OR join OR joins OR joining OR joined OR enrol* OR recruit* OR membership OR memberships OR eligib* OR entitl* OR “take part in" OR “taking part in" OR “took part in" OR "taken part in" OR "takes part in” OR enter OR entering OR entered OR enters OR register* OR registration OR registrations) OR ab: ( coverage OR cover OR covering OR covered OR covers OR participat* OR join OR joins OR joining OR joined OR enrol* OR recruit* OR membership OR memberships OR eligib* OR entitt* OR “take part in" OR “taking part in" OR "took part in" OR "taken part in" OR "takes part in" OR enter OR entering OR entered OR enters OR register* OR registration OR registrations))

41 AND 2 AND 3

14. Econlit (3 April 2009, 213 retrieved)

Outreach strategies for expanding health insurance coverage in children (Review) 
\#1 KW=(child or children or adolescent or adolescents or kid or kids or infant or infants or juvenile or juveniles or newborn or newborns or student or students or 'young people' or 'young person' or 'young persons' or teenager or teenagers or teen or teens or minor or minors or neonate or neonates or youth or youths)

\#2 KW=('health insurance' or 'health financing' or 'medical insurance' or Medicaid or 'medical scheme' or 'medical schemes' or 'community based health organization' or 'community based health organizations' or 'community-based health organization' or 'communitybased health organizations' or 'community health planning' or 'community health finance organization' or 'community health finance organizations' or 'community financing' or 'community self-financing' or 'mutual health organization' or 'mutual health organizations' or 'mutual health care' or 'mutual health association' or 'mutual health associations' or mutuelles or 'prepayment insurance organization' or 'prepayment insurance organizations' or 'cooperative medical scheme' or 'cooperative medical schemes' or 'cooperative medical system' or 'cooperative medical systems' or 'prepaid health care' or 'prepaid health plans' or 'prepaid health plan' or 'prepayment plan' or 'prepayment plans' or 'prepayment scheme' or 'prepayment schemes' or 'third-party payment' or 'third-party payer' or 'third party payment' or 'third party payer' or 'third-party payments' or 'third-party payers' or 'third party payments' or 'third party payers' or 'cost sharing' or 'risk pooling' or 'blue shield' or 'managed care programs' or 'managed care program' or 'sickness insurance')

\#3 KW= (coverage or cover or covering or covered or covers or "participat" or join or joins or joining or joined or "enrol*" or "recruit*" or membership or memberships or “eligib*” or “entitl*” or 'take part in' or 'taking part in' or 'took part in' or 'taken part in' or 'takes part in' or enter or entering or entered or enters or register or registers or registration or registrations or registered or registering) $\# 4 \# 1$ and \#2 and \#3

15. SSRN (4 June 2009, 22 retrieved)

\#1 (child OR children OR adolescent OR adolescents OR kid OR kids OR infant OR infants OR juvenile OR juveniles OR newborn OR newborns OR student OR students OR young people OR young person OR young persons OR teenager OR teenagers OR teen OR teens OR minor OR minors OR neonate OR neonates OR youth OR youths) AND health insurance

\#2 With The Exact Phrase: "medical insurance" ; "health financing" ; Medicaid ; "medical scheme" ; "medical schemes" ; "community based health organization" ; "community based health organizations" ; "community-based health organization" ; "communitybased health organizations" ; "community health planning" ; "community health finance organization" ; "community health finance organizations" ; "community financing" ; "community self-financing" ; "mutual health organization" ; "mutual health organizations" ; "mutual health care" ; "mutual health association"; "mutual health associations" ; mutuelles ; "prepayment insurance organization" ; "prepayment insurance organizations" ; "cooperative medical scheme"; "cooperative medical schemes"; "cooperative medical system"; "cooperative medical systems"; "prepaid health care"; "prepaid health plan"; "prepaid health plans"; "prepayment plan”; "prepayment plans" ; "prepayment scheme" ; "prepayment schemes" ; "third-party payment" ; "third-party payments" ; "third-party payer" ; "thirdparty payers"; "cost sharing"; "risk pooling"; "blue shield"; "managed care program"; "managed care programs"; "sickness insurance" 16. IDEAS (6 April 2009, 56 retrieved)

(child $\mid$ children $\mid$ adolescent $\mid$ kid $\mid$ infant $\mid$ juvenile $\mid$ newborn $\mid$ student $\mid$ teenager $\mid$ teen $\mid$ minor $\mid$ neonate $\mid$ youth) + (" health insurance "।" medical insurance ") + (coverage | cover | participat | enroll | eligible | entitl | register | membership)

(child $\mid$ children $\mid$ adolescent $\mid$ kid $\mid$ infant $\mid$ juvenile $\mid$ newborn $\mid$ student $\mid$ teenager $\mid$ teen $\mid$ minor $\mid$ neonate $\mid$ youth) + " health financing "| Medicaid|“ medical scheme”) + (coverage | cover | participat | enroll | eligible | entitl | register | membership)

(child | children | adolescent | kid | infant $\mid$ juvenile $\mid$ newborn $\mid$ student $\mid$ teenager $\mid$ teen $\mid$ minor $\mid$ neonate $\mid$ youth) + (" community based health organization " “" community-based health organization” ) + (coverage | cover | participat | enroll | eligible | entitl | register | membership)

(child | children | adolescent | kid | infant $\mid$ juvenile $\mid$ newborn $\mid$ student $\mid$ teenager $\mid$ teen $\mid$ minor $\mid$ neonate| youth) + (" community health planning " “ community health finance organization ”) + (coverage $\mid$ cover $\mid$ participat $\mid$ enroll $\mid$ eligible $\mid$ entitl | register $\mid$ membership)

(child | children | adolescent | kid | infant | juvenile | newborn | student | teenager | teen | minor | neonate| youth) + (" community financing”|" community self-financing”) + (coverage | cover | participat | enroll | eligible | entitl | register | membership)

(child | children $\mid$ adolescent $\mid$ kid $\mid$ infant $\mid$ juvenile $\mid$ newborn $\mid$ student $\mid$ teenager $\mid$ teen $\mid$ minor $\mid$ neonate $\mid$ youth) + (" mutual health organization ”|“ mutual health care” + ( coverage | cover | participat | enroll | eligible | entitl | register | membership)

(child $\mid$ children $\mid$ adolescent $\mid$ kid $\mid$ infant $\mid$ juvenile $\mid$ newborn $\mid$ student $\mid$ teenager $\mid$ teen $\mid$ minor $\mid$ neonate $\mid$ youth) + (" mutual health association ”| mutuelles) + (coverage $\mid$ cover $\mid$ participat $\mid$ enroll $\mid$ eligible $\mid$ entitl | register | membership)

(child $\mid$ children $\mid$ adolescent $\mid$ kid $\mid$ infant $\mid$ juvenile $\mid$ newborn $\mid$ student $\mid$ teenager $\mid$ teen $\mid$ minor $\mid$ neonate $\mid$ youth) + (" prepayment insurance organization ” |? “cooperative medical scheme”) + (coverage | cover | participat | enroll | eligible | entitl | register | membership) (child | children | adolescent | kid | infant | juvenile | newborn | student | teenager | teen | minor | neonate| youth) + (“ cooperative medical system " | " prepaid health care ") + (coverage | cover | participat | enroll | eligible | entitl | register | membership) (child | children | adolescent | kid | infant | juvenile | newborn | student | teenager | teen | minor | neonate| youth) + (" prepaid health plan "| " prepayment plan ") + (coverage | cover | participat | enroll | eligible | entitl | register | membership) 
(child | children | adolescent $\mid$ kid $\mid$ infant $\mid$ juvenile $\mid$ newborn $\mid$ student $\mid$ teenager $\mid$ teen $\mid$ minor $\mid$ neonate $\mid$ youth) + (" prepayment scheme "| "third-party payment") + (coverage | cover | participat | enroll | eligible | entitl | register | membership)

(child | children $\mid$ adolescent $\mid$ kid $\mid$ infant $\mid$ juvenile $\mid$ newborn $\mid$ student $\mid$ teenager $\mid$ teen $\mid$ minor $\mid$ neonate $\mid$ youth $)+$ (" third-party payer" | "third party payment") + (coverage | cover | participat | enroll | eligible | entitl | register | membership)

(child | children | adolescent | kid | infant | juvenile | newborn | student | teenager | teen | minor | neonate| youth) + (“ third party payer "|“ cost sharing”) + (coverage | cover | participat | enroll | eligible | entitl | register | membership)

(child | children | adolescent | kid | infant | juvenile | newborn | student | teenager | teen | minor | neonate| youth) + (" risk pooling "| "blue shield") + (coverage | cover | participat | enroll | eligible | entitl | register | membership)

(child | children | adolescent | kid | infant | juvenile | newborn | student | teenager | teen | minor | neonate| youth) + (" managed care program " | "sickness insurance ”) + (coverage | cover | participat | enroll | eligible | entitl | register | membership)

17. ELDIS (11 April 2009, 916 retrieved)

1 child OR children OR adolescent OR adolescents OR minor OR minors OR infant OR infants OR student OR students OR kid OR kids OR teenager OR teenagers OR teen OR teens OR youth OR youths OR juvenile OR juveniles OR newborn OR newborns OR neonate OR neonates OR young people OR young person OR young persons

2 health insurance OR health financing OR medical insurance OR Medicaid OR medical scheme OR medical schemes OR community based health organization OR community based health organizations OR community-based health organization OR communitybased health organizations OR community health planning OR community health finance organization OR community health finance organizations OR community financing OR community self-financing OR mutual health organization OR mutual health organizations OR mutual health care OR mutual health association OR mutual health associations OR mutuelles OR prepayment insurance organization OR prepayment insurance organizations OR cooperative medical scheme OR cooperative medical schemes OR cooperative medical system OR cooperative medical systems OR prepaid health care OR prepaid health plan OR prepaid health plans OR prepayment plan OR prepayment plans OR prepayment scheme OR prepayment schemes OR third-party payment OR thirdparty payer OR third party payment OR third party payer OR third-party payments OR third-party payers OR third party payments OR third party payers OR cost sharing OR risk pooling OR blue shield OR managed care program OR managed care programs OR sickness insurance

31 AND 2

18. BLDS (4 April 2009, 10 retrieved)

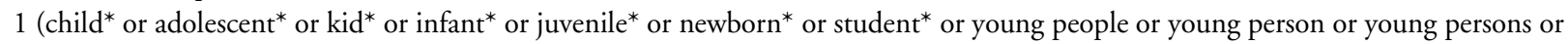
teen* or minor* or neonate* or youth*) in All fields

2 (health insurance or health financing or medical insurance or Medicaid or medical scheme or medical schemes or community based health organization or community based health organizations or community-based health organization or community-based health organizations or community health planning or community health finance organization or community health finance organizations or community financing or community self-financing or mutual health organization or mutual health organizations or mutual health care or mutual health association or mutual health associations or mutuelles or prepayment insurance organization or prepayment insurance organizations or cooperative medical scheme or cooperative medical schemes or cooperative medical system or cooperative medical systems or prepaid health care or prepaid health plans or prepaid health plan or prepayment plan or prepayment plans or prepayment scheme or prepayment schemes or third-party payment or third-party payer or third party payment or third party payer or third-party payments or third-party payers or third party payments or third party payers or cost sharing or risk pooling or blue shield or managed care programs or managed care program or sickness insurance): in All fields

31 AND 2

19. OpenSIGLE (12 April 2009, 1 retrieved)

1 (child OR children OR adolescent* OR minor OR minors OR infant OR infants OR student OR students OR kid OR kids OR teenager* OR teen OR teens OR youth OR youths OR juvenile OR juveniles OR newborn OR newborns OR neonate OR neonates OR “young people" OR “young person" OR “young persons") OR (abstract:child OR children OR adolescent* OR minor OR minors OR infant OR infants OR student OR students OR kid OR kids OR teenager* OR teen OR teens OR youth OR youths OR juvenile OR juveniles OR newborn OR newborns OR neonate OR neonates OR “young people” OR “young person” OR “young persons”) OR (title:child OR children OR adolescent* OR minor OR minors OR infant OR infants OR student OR students OR kid OR kids OR teenager* OR teen OR teens OR youth OR youths OR juvenile OR juveniles OR newborn OR newborns OR neonate OR neonates OR "young people" OR “young person" OR "young persons")

2 ("health insurance" OR "health financing" OR "medical insurance" OR Medicaid OR "medical scheme" OR "medical schemes" OR "community based health organization" OR "community based health organizations" OR "community-based health organization" OR "community-based health organizations" OR "community health planning" OR "community health finance organization" OR "community health finance organizations" OR "community financing" OR "community self-financing" OR "mutual health organi- 
zation" OR "mutual health organizations" OR "mutual health care" OR "mutual health association" OR "mutual health associations" OR mutuelles OR "prepayment insurance organization" OR "prepayment insurance organizations" OR "cooperative medical scheme" OR "cooperative medical schemes" OR "cooperative medical system" OR "cooperative medical systems" OR "prepaid health care" OR "prepaid health plan" OR "prepaid health plans" OR "prepayment plan" OR "prepayment plans" OR "prepayment scheme" OR "prepayment schemes" OR "third-party payment" OR "third-party payer" OR "third party payment" OR "third party payer" OR "thirdparty payments" OR "third-party payers" OR "third party payments" OR "third party payers" OR "cost sharing” OR "risk pooling” OR "blue shield" OR "managed care program" OR "managed care programs" OR "sickness insurance") OR (abstract:"health insurance" OR "health financing" OR "medical insurance" OR Medicaid OR "medical scheme" OR "medical schemes" OR "community based health organization" OR "community based health organizations" OR "community-based health organization" OR "communitybased health organizations" OR "community health planning" OR "community health finance organization" OR "community health finance organizations" OR "community financing" OR "community self-financing” OR "mutual health organization" OR "mutual health organizations" OR "mutual health care" OR "mutual health association" OR "mutual health associations" OR mutuelles OR "prepayment insurance organization" OR "prepayment insurance organizations" OR "cooperative medical scheme" OR "cooperative medical schemes" OR "cooperative medical system" OR "cooperative medical systems" OR "prepaid health care" OR "prepaid health plan" OR "prepaid health plans" OR "prepayment plan" OR "prepayment plans" OR "prepayment scheme" OR "prepayment schemes" OR "third-party payment" OR "third-party payer" OR "third party payment" OR "third party payer" OR "third-party payments" OR "third-party payers" OR "third party payments" OR "third party payers" OR "cost sharing” OR "risk pooling” OR "blue shield” OR "managed care program" OR "managed care programs" OR "sickness insurance") OR (title:"health insurance" OR "health financing" OR "medical insurance" OR Medicaid OR "medical scheme" OR "medical schemes" OR "community based health organization" OR "community based health organizations" OR "community-based health organization" OR "community-based health organizations" OR "community health planning" OR "community health finance organization" OR "community health finance organizations" OR "community financing” OR "community self-financing” OR "mutual health organization" OR "mutual health organizations" OR "mutual health care" OR "mutual health association" OR "mutual health associations" OR mutuelles OR "prepayment insurance organization" OR "prepayment insurance organizations" OR "cooperative medical scheme" OR "cooperative medical schemes" OR "cooperative medical system" OR "cooperative medical systems" OR "prepaid health care" OR "prepaid health plan" OR "prepaid health plans" OR "prepayment plan" OR "prepayment plans" OR "prepayment scheme” OR "prepayment schemes" OR "third-party payment" OR "third-party payer" OR "third party payment" OR "third party payer" OR "third-party payments" OR "third-party payers" OR "third party payments" OR "third party payers" OR “cost sharing” OR “risk pooling” OR "blue shield” OR "managed care program” OR "managed care programs" OR "sickness insurance")

3 (cover* OR participat* OR join OR joins OR joining OR joined OR enrol* OR recruit* OR membership* OR enter OR entering OR entered OR enters OR eligib* OR entitt* OR "take part in" OR "taking part in" OR "took part in" OR "taken part in" OR "takes part in" OR regist*) OR (abstract:cover* OR participat* OR join OR joins OR joining OR joined OR enrol* OR recruit* OR membership* OR enter OR entering OR entered OR enters OR eligib* OR entitl* OR “take part in" OR "taking part in" OR "took part in" OR "taken part in" OR “takes part in" OR regist*) OR (title:cover* OR participat* OR join OR joins OR joining OR joined OR enrol* OR recruit* OR membership* OR enter OR entering OR entered OR enters OR eligib* OR entitl* OR "take part in" OR "taking part in" OR "took part in" OR "taken part in" OR "takes part in" OR regist*)

41 AND 2 AND 3

20. NTIS (3 April 2009, 549 retrieved)

$\# 1 \mathrm{KW}=$ (child or children or adolescent or adolescents or kid or kids or infant or infants or juvenile or juveniles or newborn or newborns or student or students or 'young people' or 'young person' or 'young persons' or teenager or teenagers or teen or teens or minor or minors or neonate or neonates or youth or youths)

\#2 KW=('health insurance' or 'health financing' or 'medical insurance' or Medicaid or 'medical scheme' or 'medical schemes' or 'community based health organization' or 'community based health organizations' or 'community-based health organization' or 'communitybased health organizations' or 'community health planning' or 'community health finance organization' or 'community health finance organizations' or 'community financing' or 'community self-financing' or 'mutual health organization' or 'mutual health organizations' or 'mutual health care' or 'mutual health association' or 'mutual health associations' or mutuelles or 'prepayment insurance organization' or 'prepayment insurance organizations' or 'cooperative medical scheme' or 'cooperative medical schemes' or 'cooperative medical system' or 'cooperative medical systems' or 'prepaid health care' or 'prepaid health plans' or 'prepaid health plan' or 'prepayment plan' or 'prepayment plans' or 'prepayment scheme' or 'prepayment schemes' or 'third-party payment' or 'third-party payer' or 'third party payment' or 'third party payer' or 'third-party payments' or 'third-party payers' or 'third party payments' or 'third party payers' or 'cost sharing' or 'risk pooling' or 'blue shield' or 'managed care programs' or 'managed care program' or 'sickness insurance')

Outreach strategies for expanding health insurance coverage in children (Review) 
\#3 KW=(coverage or cover or covering or covered or covers or "participat" or join or joins or joining or joined or "enrol*" or "recruit*" or membership or memberships or "eligib*” or “entitl*” or 'take part in' or 'taking part in' or 'took part in' or 'taken part in' or 'takes part in' or enter or entering or entered or enters or register or registers or registration or registrations or registered or registering)

$\# 4 \# 1$ and \#2 and \#3

21. ProQuest Dissertation \& Theses Database (5 April 2009, 644 retrieved)

(child OR children OR adolescent OR adolescents OR minor OR minors OR infant OR infants OR student OR students OR kid OR kids OR teenager OR teenagers OR teen OR teens OR youth OR youths OR juvenile OR juveniles OR newborn OR newborns OR neonate OR neonates OR young people OR young person OR young persons) AND (insurance OR health insurance OR health financing OR medical insurance OR Medicaid OR medical scheme OR medical schemes OR "community based health organization" OR "community based health organizations" OR "community-based health organization" OR "community-based health organizations" OR "community health planning" OR "community health finance organization" OR "community health finance organizations" OR community financing OR community self-financing OR "mutual health organization" OR "mutual health organizations" OR "mutual health care" OR "mutual health association" OR "mutual health associations" OR mutuelles OR "prepayment insurance organization" OR "prepayment insurance organizations" OR "cooperative medical scheme" OR "cooperative medical schemes" OR "cooperative medical system" OR "cooperative medical systems" OR "prepaid health care" OR "prepaid health plan" OR "prepaid health plans" OR prepayment plan OR prepayment plans OR prepayment scheme OR prepayment schemes OR third-party payment OR third-party payer OR "third party payment" OR "third party payer" OR third-party payments OR third-party payers OR "third party payments" OR "third party payers" OR cost sharing OR risk pooling OR blue shield OR "managed care program" OR "managed care programs" OR sickness insurance) AND (cover* OR participat* OR join OR joins OR joining OR joined OR enrol* OR recruit* OR membership OR memberships OR enter OR entering OR entered OR enters OR eligib* OR entitl* OR "take part in" OR "taking part in" OR "took part in" OR "taken part in" OR "takes part in" OR regist*)

22. ISI Proceedings (5 April 2009, 1507 retrieved)

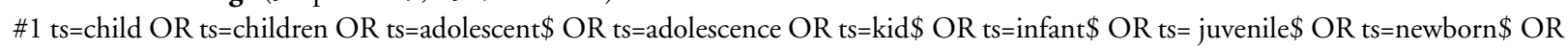

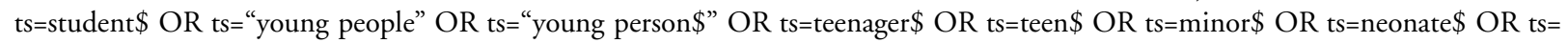
youth\$

\#2 ts="health insurance" OR ts="health financing" OR ts="medical insurance" OR ts=Medicaid OR ts="medical scheme\$” OR ts= "community based health organization\$" OR ts="community-based health organization\$" OR ts="community health planning" OR $\mathrm{ts}=$ "community health finance organization\$" OR ts="community financing" OR ts="community self-financing" OR ts="mutual health organization\$" OR ts="mutual health care" OR ts="mutual health association\$” OR ts=mutuelles OR ts="prepayment insurance Organization\$" OR ts="cooperative medical scheme\$” OR ts="cooperative medical system\$” OR ts="prepaid health care" OR ts= "prepaid health plan\$” OR ts="prepayment plan\$” OR ts="prepayment scheme\$” OR ts="third-party payment\$” OR ts="third-party payer\$" OR ts="third party payment\$" OR ts="third party payer\$" OR ts="cost sharing" OR ts="risk pooling" OR ts="blue shield" OR ts="managed care program\$" OR ts="sickness insurance"

\#3 ts=cover* OR ts=participat* OR ts=join* OR ts=enrol* OR ts=recruit* OR ts=membership\$ OR ts=eligib* OR ts=entitl* OR ts= "take part in" OR ts="taking part in" OR ts="took part in" OR ts="taken part in" OR ts="takes part in" OR ts="enter*” OR ts= "register" "

\#4 \#1 AND \#2 AND \#3

23. PAHO Library Catalogue (6 January 2010, 66 retrieved)

1 children [Subject descriptor] and health insurance [Subject descriptor]

2 children [Subject descriptor] and health insurance or health financing or medical insurance or Medicaid or medical scheme or medical schemes or community based health organization or community based health organizations or community-based health organization or community-based health organizations or community health planning or community health finance organization or community health finance organizations or community financing or community self-financing or mutual health organization or mutual health organizations or mutual health care or mutual health association or mutual health associations or mutuelles or prepayment insurance organization or prepayment insurance organizations or cooperative medical scheme or cooperative medical schemes or cooperative medical system or cooperative medical systems or prepaid health care or prepaid health plans or prepaid health plan or prepayment plan or prepayment plans or prepayment scheme or prepayment schemes or third-party payment or third-party payer or third party payment or third party payer or third-party payments or third-party payers or third party payments or third party payers or cost sharing or risk pooling or blue shield or managed care programs or managed care program or sickness insurance [Words]

3 child or adolescent or kid or infant or juvenile or newborn or student or young people or young person or teen or minor or neonate or youth [Words] and health insurance or health financing or medical insurance or Medicaid or medical scheme or medical schemes or community based health organization or community based health organizations or community-based health organization or community-based health organizations or community health planning or community health finance organization or community 
health finance organizations or community financing or community self-financing or mutual health organization or mutual health organizations or mutual health care or mutual health association or mutual health associations or mutuelles or prepayment insurance organization or prepayment insurance organizations or cooperative medical scheme or cooperative medical schemes or cooperative medical system or cooperative medical systems or prepaid health care or prepaid health plans or prepaid health plan or prepayment plan or prepayment plans or prepayment scheme or prepayment schemes or third-party payment or third-party payer or third party payment or third party payer or third-party payments or third-party payers or third party payments or third party payers or cost sharing or risk pooling or blue shield or managed care programs or managed care program or sickness insurance [Words]

4 health and insurance and coverage

51 OR 2 OR 3 OR 4

24. African Index Medicus (5 April 2009, 3 retrieved)

1 (child or children or adolescent or adolescents or kid or kids or infant or infants or juvenile or juveniles or newborn or newborns or student or students or (young and people) or (young and person) or (young and persons) or teenager or teenagers or teen or teens or minor or minors or neonate or neonates or youth or youths) [Key Word]

2 ((health and insurance) or (health and financing) or (medical and insurance) or Medicaid or (medical and scheme) or (medical and schemes) or (community and based and health and organization) or (community and based and health and organizations) or (community and health and planning) or (community and health and finance and organization) or (community and health and finance and organizations) or (community and financing) or (community and self-financing) or (mutual and health and organization) or (mutual and health and organizations) or (mutual and health and care) or (mutual and health and association) or (mutual and health and associations) or mutuelles or (prepayment and insurance and organization) or (prepayment and insurance and organizations) or (cooperative and medical and scheme) or (cooperative and medical and schemes) or (cooperative and medical and system) or (cooperative and medical and systems) or (prepaid and health and care) or (prepaid and health and plans) or (prepaid and health and plan) or (prepayment and plan) or (prepayment and plans) or (prepayment and scheme) or (prepayment and schemes) or (third and party and payment) or (third and party and payer) or (third and party and payments) or (third and party and payers) or (cost and sharing) or (risk and pooling) or (blue and shield) or (managed and care and programs) or (managed and care and program) or (sickness and insurance)) [Key Word]

31 and 2

25. HERDIN NeON Database (5 April 2009, 0 retrieved)

1 (child $\$$ or adolescent $\$$ or kid $\$$ or infant $\$$ or juvenile $\$$ or newborn $\$$ or student $\$$ or young people or young person $\$$ or teen $\$$ or minor $\$$ or neonate\$ or youth\$): Keywords (all fields)

2 (health insurance or health financing or medical insurance or Medicaid or medical scheme or medical schemes or community based health organization or community based health organizations or community-based health organization or community-based health organizations or community health planning or community health finance organization or community health finance organizations or community financing or community self-financing or mutual health organization or mutual health organizations or mutual health care or mutual health association or mutual health associations or mutuelles or prepayment insurance organization or prepayment insurance organizations or cooperative medical scheme or cooperative medical schemes or cooperative medical system or cooperative medical systems or prepaid health care or prepaid health plans or prepaid health plan or prepayment plan or prepayment plans or prepayment scheme or prepayment schemes or third-party payment or third-party payer or third party payment or third party payer or third-party payments or third-party payers or third party payments or third party payers or cost sharing or risk pooling or blue shield or managed care programs or managed care program or sickness insurance): Keywords (all fields)

31 AND 2

26. Thai Index Medicus (6 January 2010, 205 retrieved)

1 health AND insurance AND coverage

2 health AND insurance AND children

31 OR 2

27. Scientific and Technical Egyptian Bibliographic Database (STEB) (5 April 2009, 36 retrieved)

1 keyword: (child or children or adolescent or adolescents or kid or kids or infant or infants or juvenile or juveniles or newborn or newborns or student or students or young people or young person or young persons or teenager or teenagers or teen or teens or minor or minors or neonate or neonates or youth or youths)

2 keyword: (health insurance or health financing or medical insurance or Medicaid or medical scheme or medical schemes or community based health organization or community based health organizations or community-based health organization or communitybased health organizations or community health planning or community health finance organization or community health finance organizations or community financing or community self-financing or mutual health organization or mutual health organizations or mutual health care or mutual health association or mutual health associations or mutuelles or prepayment insurance organization or 
prepayment insurance organizations or cooperative medical scheme or cooperative medical schemes or cooperative medical system or cooperative medical systems or prepaid health care or prepaid health plans or prepaid health plan or prepayment plan or prepayment plans or prepayment scheme or prepayment schemes or third-party payment or third-party payer or third party payment or third party payer or third-party payments or third-party payers or third party payments or third party payers or cost sharing or risk pooling or blue shield or managed care programs or managed care program or sickness insurance)

31 AND 2

28. IndMED (5 April 2009, 16 retrieved)

1 (child $\$$ or adolescent $\$$ or kid $\$$ or infant $\$$ or juvenile $\$$ or newborn $\$$ or student $\$$ or young people or young person $\$$ or teen $\$$ or minor $\$$ or neonate\$ or youth\$): in Keywords

2 (health insurance or health financing or medical insurance or Medicaid or medical scheme or medical schemes or community based health organization or community based health organizations or community-based health organization or community-based health organizations or community health planning or community health finance organization or community health finance organizations or community financing or community self-financing or mutual health organization or mutual health organizations or mutual health care or mutual health association or mutual health associations or mutuelles or prepayment insurance organization or prepayment insurance organizations or cooperative medical scheme or cooperative medical schemes or cooperative medical system or cooperative medical systems or prepaid health care or prepaid health plans or prepaid health plan or prepayment plan or prepayment plans or prepayment scheme or prepayment schemes or third-party payment or third-party payer or third party payment or third party payer or third-party payments or third-party payers or third party payments or third party payers or cost sharing or risk pooling or blue shield or managed care programs or managed care program or sickness insurance): in Keywords

31 AND 2

29. LILACS (30 June 2009, 307 retrieved)

(health $\$$ and insurance $\$$ ) or (medical $\$$ and insurance $\$$ ) or (insurance $\$$ and program $\$$ ) or (seguro and medico $\$$ ) or (seguro and salud) or (seguro and saude) [Palavras] and child or children or nino $\$$ or crianca or infant or infants or infantil or lactante or lactente or newborn or (recien and nacido\$) or (recem and nascido\$) or neonat $\$$ or baby or babies or kid or kids or toddler\$ [Palavras] and ((Pt RANDOMIZED CONTROLLED TRIAL OR Pt CONTROLLED CLINICAL TRIAL OR Mh RANDOMIZED CONTROLLED TRIALS OR Mh RANDOM ALLOCATION OR Mh DOUBLE-BLIND METHOD OR Mh SINGLE-BLIND METHOD OR Pt MULTICENTER STUDY) OR ((time and series) or random $\$$ or intervention $\$$ or control\$ or evaluat $\$$ or effect $\$$ or compare $\$$ or chang\$ or experiment $\$$ or impact) or ( tw ensaio or tw ensayo or tw trial) and (tw azar or tw acaso or tw placebo or tw control\$ or tw aleat $\$$ or tw random $\$$ or (tw duplo and tw cego) or (tw doble and tw ciego) or (tw double and tw blind)) and tw clinic\$)) AND NOT ((CT ANIMALS OR MH ANIMALS OR CT RABBITS OR CT MICE OR MH RATS OR MH PRIMATES OR MH DOGS OR MH RABBITS OR MH SWINE) AND NOT (CT HUMAN AND CT ANIMALS)) [Palavras]

30. ChinaNational Knowledge Infrastructure (CHKD-CNKI) (6 April 2009, 288 retrieved)

See http://epocoslo.cochrane.org/sites/epocoslo.cochrane.org/files/uploads/Chinese\%20databases\%20search\%20strategies.pdf

31. Chinese Medicine Premier (Wanfang Data) (9 April 2009, 0 retrieved)

See http://epocoslo.cochrane.org/sites/epocoslo.cochrane.org/files/uploads/Chinese\%20databases\%20search\%20strategies.pdf

32. ID21 (4 April 2009, 69 retrieved)

1 child* or adolescent* or kid* or infant* or juvenile* or newborn* or student* or young people or young person or young persons or teen* or minor* or neonate* or youth*

2 health insurance or health financing or medical insurance or Medicaid or medical scheme or medical schemes or community based health organization or community based health organizations or community-based health organization or community-based health organizations or community health planning or community health finance organization or community health finance organizations or community financing or community self-financing or mutual health organization or mutual health organizations or mutual health care or mutual health association or mutual health associations or mutuelles or prepayment insurance organization or prepayment insurance organizations or cooperative medical scheme or cooperative medical schemes or cooperative medical system or cooperative medical systems or prepaid health care or prepaid health plans or prepaid health plan or prepayment plan or prepayment plans or prepayment scheme or prepayment schemes or third-party payment or third-party payer or third party payment or third party payer or third-party payments or third-party payers or third party payments or third party payers or cost sharing or risk pooling or blue shield or managed care programs or managed care program or sickness insurance

31 and 2

Outreach strategies for expanding health insurance coverage in children (Review) 


\section{Appendix 2. Criteria for appraising risk of bias}

For RCTs, CCTs and CBAs the criteria are:

- Was the allocation sequence adequately generated? Score "yes" if a random component in the sequence generation process is described (e.g. referring to a random number table). Score "no" when a non-random method is used (e.g. performed by date of admission). CCTs and CBAs should be scored "no". Score "unclear" if not specified in the paper.

- Was the allocation adequately concealed? Score "yes" if the unit of allocation was by institution, team or professional and allocation was performed on all units at the start of the study; or if the unit of allocation was by patient or episode of care and there was some form of centralised randomisation scheme, an on-site computer system or sealed opaque envelopes were used. CBAs should be scored "no". Score "unclear" if not specified in the paper.

- Were baseline outcome measurements similar? Score "yes" if performance or patient outcomes were measured prior to the intervention, and no important differences were present across study groups. In RCTs, score "yes" if imbalanced but appropriate adjusted analysis was performed (e.g. analysis of covariance). Score "no" if important differences were present and not adjusted for in analysis. If RCTs have no baseline measure of outcome, score "unclear".

- Were baseline characteristics similar? Score "yes" if baseline characteristics of the study and control providers are reported and similar. Score "unclear" if it is not clear in the paper (e.g. characteristics are mentioned in text but no data were presented). Score "no" if there is no report of characteristics in text or tables or if there are differences between control and intervention providers. Note that in some cases imbalance in patient characteristics may be due to recruitment bias whereby the provider was responsible for recruiting patients into the trial.

- Were incomplete outcome data adequately addressed? Score "yes" if missing outcome measures were unlikely to bias the results (e.g. the proportion of missing data was similar in the intervention and control groups or the proportion of missing data was less than the effect size, i.e. unlikely to overturn the study result). Score "no" if missing outcome data were likely to bias the results. Score "unclear" if not specified in the paper. (Do not assume $100 \%$ follow up unless stated explicitly).

- Was knowledge of the allocated interventions adequately prevented during the study? Score "yes" if the authors state explicitly that the primary outcome variables were assessed blindly, or the outcomes are objective. Primary outcomes are those variables that correspond to the primary hypothesis or question as defined by the authors. Score "no" if the outcomes were not assessed blindly. Score "unclear" if not specified in the paper.

- Was the study adequately protected against contamination? Score "yes" if allocation was by community, institution or practice and it is unlikely that the control group received the intervention. Score "no" if it is likely that the control group received the intervention (e.g. if patients rather than professionals were randomised). Score "unclear" if professionals were allocated within a clinic or practice and it is possible that communication between intervention and control professionals could have occurred (e.g. physicians within practices were allocated to intervention or control).

- Was the study free from selective outcome reporting? Score "yes" if there is no evidence that outcomes were selectively reported (e.g. all relevant outcomes in the methods section are reported in the results section). Score "no" if some important outcomes are subsequently omitted from the results. Score "unclear" if not specified in the paper.

- Was the study free from other risks of bias? Score "yes" if there is no evidence of other risk of biases.

For ITS studies, the criteria are:

- Was the intervention independent of other changes? Score "yes" if there are compelling arguments that the intervention occurred independently of other changes over time and the outcome was not influenced by other confounding variables/historical events during the study period. If events/variables identified, note what they are. Score "no" if it is reported that the intervention was not independent of other changes in time.

- Was the shape of the intervention effect pre-specified? Score "yes" if the point of analysis is the point of intervention OR a rational explanation for the shape of intervention effect was given by the author(s). Where appropriate, this should include an explanation if the point of analysis is NOT the point of intervention. Score "no" if it is clear that the condition above is not met.

- Was the intervention unlikely to affect data collection? Score "yes" if reported that the intervention itself was unlikely to affect data collection (for example, sources and methods of data collection were the same before and after the intervention). Score "no" if the intervention itself was likely to affect data collection (for example, any change in source or method of data collection reported).

- Was knowledge of the allocated interventions adequately prevented during the study? Score "yes" if the authors state explicitly that the primary outcome variables were assessed blindly, or the outcomes are objective. Primary outcomes are those variables that correspond to the primary hypothesis or question as defined by the authors. Score "no" if the outcomes were not assessed blindly. Score "unclear" if not specified in the paper.

- Were incomplete outcome data adequately addressed? Score "yes" if missing outcome measures were unlikely to bias the results (e.g. the proportion of missing data was similar in the pre- and post-intervention periods or the proportion of missing data was less 
than the effect size, i.e. unlikely to overturn the study result). Score "no" if missing outcome data were likely to bias the results. Score "unclear" if not specified in the paper. (Do not assume 100\% follow up unless stated explicitly).

- Was the study free from selective outcome reporting? Score "yes" if there is no evidence that outcomes were selectively reported (e.g. all relevant outcomes in the methods section are reported in the results section). Score "no" if some important outcomes are subsequently omitted from the results. Score "unclear" if not specified in the paper.

- Was the study free from other risks of bias? Score "yes" if there is no evidence of other risk of biases.

\section{H I S T O R Y}

Protocol first published: Issue 1, 2010

Review first published: Issue 8, 2010

\section{CONTRIBUTIONSOFAUTHORS}

All review authors have contributed to the production of the review. Qingyue Meng drafted the protocol. Beibei Yuan and Liying Jia amended it with comments from all other co-authors. Beibei Yuan and Liying Jia applied the inclusion criteria, assessed the risk of bias and extracted data for the included studies. Beibei Yuan and Liying Jia prepared the report and other review authors commented on it.

\section{DECLARATIONS OF INTEREST}

There are no conflicts of interest.

\section{SOURCES OF SUPPORT}

\section{Internal sources}

- No sources of support supplied

\section{External sources}

- The Alliance for Health Policy and Systems Research, World Health Organization, Not specified.

\section{DIFFERENCES BETWEEN PROTOCOLANDREVIEW}

We did not search the HIMC database because it is inaccessible in Shandong University, Liverpool School of Topical Medicine and the University of Oslo. 


\section{N D EX TERMS}

\section{Medical Subject Headings (MeSH)}

Adolescent; Documentation [methods]; Emergency Service, Hospital; Insurance Coverage [ ${ }^{*}$ organization \& administration; statistics \& numerical data]; Insurance, Health [*organization \& administration; statistics \& numerical data]; Latin America [ethnology]; Medically Uninsured [statistics \& numerical data]; Randomized Controlled Trials as Topic; United States

\section{MeSH check words}

Child; Humans 